Pure and Applied Mathematics Quarterly

Volume 1, Number 3

(Special Issue: In Memory of

Armand Borel, Part 2 of 3 )

449-478, 2005

\title{
Rationality of Renormalized Chern Classes
}

\author{
D. Burns and J.-S. Ryu \\ In memory of Armand Borel, and especially \\ his support of young scientists.
}

\begin{abstract}
Renormalized Chern classes $\tilde{c}_{2}, \ldots, \tilde{c}_{n}$ for a compact, connected, complex manifold $X$ with smooth, strictly pseudoconvex boundary $M$ were defined in [7]. A Chern-Simons type invariant was also defined in [6] for a compact, strictly pseudoconvex $C R$-manifold $M$ of dimension three, and a Gauss-Bonnet theorem relating the two in [7]. We show here that if $M$ is spherical, and $P\left(\tilde{c}_{2}, \ldots, \tilde{c}_{n}\right)$ is a polynomial with rational coefficients in $\tilde{c}_{2}, \ldots, \tilde{c}_{n}$, then the corresponding Chern class $P(X)=P\left(\tilde{c}_{2}, \ldots, \tilde{c}_{n}\right)$ defines a class in $H^{*}(X, M ; \mathbb{Q})$. The proof is based on the multi-valued Hartogs theorem of [5] and the exponents of monodromy for a development map.
\end{abstract}

\section{INTRODUCTION}

A characteristic number $\mu(M)$ for three dimensional compact strongly pseudoconvex $C R$ manifolds $M$ with $C R$ sub-bundle of the tangent bundle a torsion complex line bundle, was introduced in [6], constructed analogously to the ChernSimons invariant $\alpha(Y,[g]) \in \mathbb{R} / \mathbb{Z}$ for the conformal class $[g]$ of a Riemannian metric $g$ on a compact three manifold $Y$. Several examples were computed in [6], and for all those $M$ which were spherical, i.e., locally $C R$ equivalent to the standard sphere, it was found that $\mu(M)$, which is a priori a real number, is rational.

In [7] renormalized Chern forms were described for $X$ a compact complex manifold with strictly pseudoconvex boundary $\partial X=M$. Roughly speaking, these are forms computed in a class of natural complete Kähler metrics on $X$

Received May 21, 2005.

Supported in part by NSF grant DMS-0104047. 
which includes the Bergman metric, the Kähler-Einstein metric, etc. In [7] only the Kähler-Einstein metric was used explicitly. In effect, one uses the asymptotic complex hyperbolicity of such metrics to show the convergence of characteristic number integrals for those characteristic forms which would vanish identically on the complex ball in the complex hyperbolic metric. This gives, in general, classes whose integrals in the top dimension are again only real numbers. In the case $\operatorname{dim}_{\mathbb{C}} X=2$, a kind of Gauss-Bonnet formula was shown in [7] relating the integral of the renormalized Chern-form $\tilde{c}_{2}$ with the Euler characteristic of $X$, $\mu(M)$, and a topological (rational) characteristic number:

$$
\int_{X} \tilde{c}_{2}=\chi(X)-\frac{1}{3} \bar{c}_{1}^{2}+\mu(M)
$$

Here $\bar{c}_{1} \in H^{2}(X, M ; \mathbb{Q})$ is the first Chern class of the canonical line bundle $\Omega_{X}^{2}$ corresponding to a topological trivialization near the boundary of some power of $\Omega_{X}^{2}$. In particular, the renormalized Chern number $\int_{X} \tilde{c}_{2} \in \mathbb{Q}$ if the boundary $M$ is any of the spherical examples computed in [6]. Our purpose here is to show that this rationality is always true for spherical $M$ and for any renormalized Chern number.

More specifically, let $X$ be as above, with $\operatorname{dim}_{\mathbb{C}} X=n$, and let $g$ be a Kähler metric on $X$ of the type allowed in [7], that is, asymptotically complete KählerEinstein at the boundary. (In [7] only complete Kähler-Einstein metrics were used. In section 3 . below, however, we check that the techniques of [7] allow one to extend the theory of [7] to metrics which are only sufficiently close asymptotically to such a Kähler-Einstein metric at the boundary.) Let $\tilde{c}_{2}, \ldots, \tilde{c}_{n}$ be the renormalized Chern forms for this metric, and let $P=P\left(\tilde{c}_{2}, \ldots, \tilde{c}_{n}\right)$ be a polynomial with rational coefficients in the renormalized Chern forms, a form of degree exactly $2 n$.

Theorem 1.1. (Main Theorem) $X, P, g$ as above. Then $P(X)=\int_{X} P\left(\tilde{c}_{2}, \ldots, \tilde{c}_{n}\right)$ is rational.

In fact, a more precise theorem is proved. When $M$ is spherical, one may choose a metric on $X$ with which to compute the $\tilde{c}_{j}, j=2, \ldots, n$ for which these forms vanish near the boundary $M$. These forms define classes $\left[\tilde{c}_{j}\right] \in H^{2 j}(X, M ; \mathbb{R})$ which are independent of the choice of metric (within a class of metrics). The more precise statement is the following

Theorem 1.2. The classes $\left[\tilde{c}_{j}\right], j=2, \ldots, n$, are in $H^{2 j}(X, M ; \mathbb{Q})$.

The Main Theorem follows immediately from Theorem 1.2.

We use the rest of this introduction to sketch the proof of Theorem 1.2 and to outline the contents of the rest of the paper. Let $E$ be the maximal compact 
subvariety contained in $X$. Using the fact that $M$ is spherical and a development map technique in [5], we can replace the metric $g$ by another complete Kähler metric $\tilde{g}$ which is locally complex hyperbolic outside any open neighborhood of $E$, and a transgression formula as in [7] allows one to replace the renormalized Chern forms in the metric $g$ by those in the new metric $\tilde{g}$, and in such a metric the renormalized forms vanish identically wherever the metric is locally complex hyperbolic. Thus, if the analytic set $E$ is empty or of codimension $>1$, the metric $\tilde{g}$ can be defined everywhere on $X$ and $P(X)=0$, since its integrand vanishes. In general, the (multi-valued) developing map $D: X \backslash E \rightarrow \mathbb{B}^{n}$ which pulls back the complex hyperbolic metric $d s_{\mathrm{CH}}^{2}$ from the complex unit ball $\mathbb{B}^{n} \subset \mathbb{C}^{n}$ doesn't extend across $E$, and is singular there. Roughly speaking, in the limit, the characteristic form integral should be calculable by a kind of residue formula along $E$ taking into account the various singularities of the metric $D^{*} d s_{\mathrm{CH}}^{2}$ there. The main point of the theorem is to analyze the nature of these singularities, though we will use an indirect, geometric argument for calculating the final integrals rather than a direct residue calculation.

Two elementary examples in complex dimension one illustrate this phenomenon. Let $X=\Delta$, the unit disk, and consider the mapping $D$ from $\Delta \backslash\{0\}=$ $\Delta^{*} \ni z \rightarrow w=z^{\alpha} \in \Delta$, with $0<\alpha \in \mathbb{R}$. $D$ is, in general, multi-valued, but if we pull back a hermitian metric $g=\rho\left(|w|^{2}\right)|d w|^{2}$, the metric $D^{*} g$ is well-defined on $\Delta^{*}$ and possibly singular at the origin. Looking first at the case of $g$ the Poincaré metric $\frac{|d w|^{2}}{\left(1-|w|^{2}\right)^{2}}$ on $\Delta$ and calculating the first Chern form of $D^{*} g$ distributionally, we get that

$$
D^{*} g=\alpha^{2} \frac{|z|^{2(\alpha-1)}|d z|^{2}}{\left(1-|z|^{2 \alpha}\right)^{2}}
$$

and therefore,

$$
c_{1}\left(D^{*} g\right)=\frac{\sqrt{-1}}{2 \pi} \partial \bar{\partial} \log \frac{|z|^{2(\alpha-1)}}{\left(1-|z|^{2 \alpha}\right)^{2}}=(\alpha-1) \delta_{0}+D^{*} c_{1}(g),
$$

so that the contribution of the singularity to the integral of the distributional Chern form is $\alpha-1$, and in particular is rational if and only if the monodromy exponent is rational.

On the other hand, if we take the example $g=\frac{|d w|^{2}}{|w|^{2} \log ^{2}|w|^{2}}$, i.e., the Poincaré metric on the punctured disk $\Delta^{*}$, then $D^{*} g(w)=g(z)$ and computing distributionally, $c_{1}\left(D^{*} g\right)=D^{*} c_{1}(g)$, which is integrable, and there is no singular contribution to the distributional first Chern form.

In our case, the irreducible divisors $E_{j} \subset E$ are divided into the elliptic and parabolic divisors, the elliptic ones being generalizations of the first example above, and the parabolic divisors generalizing the second example. For the elliptic divisors, the exponents of the principal monodromy, corresponding to passing 
once around the divisor $E_{j}$, determine whether the global Chern classes are rational. We do not compute the characteristic integrals as explicitly as in the elementary case above, but use more geometric arguments to pass to carefully chosen coverings of $X$ near $E$ where we can extend the development map locally across the compact subvariety. Simple topological comparisons enable us to shift our considerations at a parabolic divisor to the higher dimensional analogue of the second example above, and a similar elementary residue calculation such as is implicit in the calculation of the second example above, and which was used previously at least as early as in Mumford's paper [23], shows that in a precise sense these divisors do not contribute to the Chern class.

Section 2 collects some needed details about renormalized Chern forms and fills in some gaps in the literature as to their dependence on approximations to the Kähler-Einstein metric. In particular, we verify that for $M$ spherical, the forms $\tilde{c}_{j}$ determine well-defined classes in $H^{2 j}(X, M ; \mathbb{R})$. In section 3 we review what we need of the development map technique of [5], in particlar, the multi-valued Hartogs theorem, and use this to pull back the complex hyperbolic metric from $\mathbb{B}^{n}$ to $X$, and thus concentrate the classes studied to neighborhoods of the maximal compact subvariety of $X$. In section 4 we analyze the principal monodromies. The irreducible divisors of $E$ are classified according to whether these monodromies are elliptic or parabolic. Composing the monodromy representation with the adjoint representation of $P S U(n, 1)$ on the complexification $\mathfrak{s u}(n, 1) \otimes \mathbb{C}$ of $\mathfrak{s u}(n, 1)$, and some reductions, we prove that the eigenvalues of the principal monodromy transformations are roots of unity. This comes from standard relations on the fundamental group and intersection matrices of curves in surfaces due to Mumford [22], though care has to be taken in "abelianizing" a non-abelian representation. Section 5 carries out a final preparatory geometric reduction of the principal monodromies, and a sharper analysis of the case of a parabolic divisor. Section 6 constructs a completion of the pull-back of the tangent bundle $T \mathbb{B}^{n}$ by the development map which is compared to the tangent bundle $T X$ by the differential of the development map. This comparison completes the proof of the Main Theorem 1.1. A final section 7 gives a brief description of an analogous problem for real conformally compact manifolds, and recent work on renormalizing volumes of manifolds like our $X$.

The authors would like to thank both the University of Michigan (JSR), Pohang University of Science and Technology and Seoul National University (DB), and the Korean Institute for Advanced Study (DB and JSR) for visitor support during the preparation of this paper. The authors would also like to thank Gang Tian for useful discussions concerning this subject at an early stage of our work. 


\section{Renormalized Chern Forms.}

In this section we recall the basics of renormalized Chern forms from [7] and extend them to cover the current situation. We first describe the class of metrics for which the renormalized Chern forms will be defined, and use the standard transgression formula to check the extent to which they are independent of the metric used within this class.

In [7], it was assumed one had a Kähler-Einstein metric $g$ on a compact complex manifold with smooth, strictly pseudoconvex boundary, and if we define the tensors

$$
K=K_{i k \bar{\ell}}^{j}=-1\left\{\delta_{i}^{j} g_{k \bar{\ell}}+\delta_{k}^{j} g_{i \bar{\ell}}\right\}
$$

and

$$
R i c c i=\frac{1}{n+1} \operatorname{Ric}_{i \bar{\ell}} \delta_{k}^{j}+\frac{1}{n+1} \operatorname{Ric}_{k \bar{\ell}} \delta_{i}^{j}
$$

and

$$
W_{i k \bar{\ell}}^{j}=R i e m-R i c c i,
$$

where $\operatorname{Riem}_{i k \bar{\ell}}^{j}$ is the (Kähler) Riemannian curvature tensor, Ric $=\operatorname{Ric}_{i \bar{\ell}}=$ Riem $_{i j \bar{\ell}}^{j}$ is the Ricci form. This leads to matrices of 2 -forms

$$
\Omega=\left(\Omega_{i}^{j}\right)=\left(\sum_{k, \bar{\ell}} \operatorname{Riem}_{i k \bar{\ell}}^{j} d z_{k} \wedge d \bar{z}_{\ell}\right),
$$

and

$$
W=\left(W_{i}^{j}\right)=\left(\sum_{k, \ell} W_{i k \ell}^{j} d z_{k} \wedge d \bar{z}_{\ell}\right)
$$

Explicitly, in [7], it was assumed that $R i c_{i \bar{j}} \equiv-(n+1) g_{i \bar{j}}$, or equivalently

$$
E:=\text { Ricci }-K=0
$$

A renormalized Chern form was then taken to be a polynomial in the power traces $\operatorname{Tr}\left(W^{j}\right), j=2, \ldots, n$, which could be expressed in terms of the Chern forms of the metric $g$. Specific renormalized characteristic forms $\tilde{c}_{j}, j=2, \ldots, n,\left(\tilde{c}_{1}=0\right)$, the renormalized Chern classes, were defined inductively by

$$
c_{j}=\tilde{c}_{j}+\sum_{i=1}^{j} a_{j, i}\left(\tilde{c}_{2}, \ldots, \tilde{c}_{j-1}\right) c_{1}^{i},
$$

where the $a_{j, i}\left(\tilde{c}_{2}, \ldots, \tilde{c}_{j-1}\right)$ are $2 j-2 i$-forms, polynomial in the already defined renormalized Chern classes. We will here check more carefully that the renormalized Chern classes may be defined in terms of approximations to a Kähler-Einstein 
metric, as in [14], and that these forms will yield the same characteristic numbers as in [7].

Let first $v_{0}$ be a volume form on $X$ which is smooth and strictly positive up to and including the boundary $M$. In local holomorphic coordinates $z_{1}, \ldots, z_{n}$ smooth to the boundary near a boundary point $z_{0} \in M$, we can write

$$
v_{0}=\rho_{0}(z) \prod_{j=1}^{n} \frac{\sqrt{-1}}{2} d z_{j} \wedge d \bar{z}_{j},
$$

where $\rho_{0}$ is smooth and strictly positive up to the boundary. Consider all volume forms $v$ of the form

$$
v=\frac{1}{(-\psi)^{n+1}} v_{0}
$$

where $\psi$ is a smooth function vanishing on $M$, with $d \psi \neq 0$ on $M$, and $\psi<0$ on the interior of $X$. The Ricci form is

$$
\operatorname{Ric}(v)=\partial \bar{\partial} \log \frac{(-\psi)^{n+1}}{\rho_{0}} .
$$

We set $g=g(v)=-\frac{1}{n+1} \operatorname{Ric}(v)$ sufficiently close to $M$ and extend it to all of $X$ as a hermitian metric on the tangent bundle $T X$. This metric is Kähler near $M$. We will say that the volume form $v$ is a Fefferman approximation if it satisfies

$$
\operatorname{Ric}(v)+(n+1) g=O\left(\psi^{n-1}\right) \text { at the boundary. }
$$

That such volume forms exist locally near $z_{0}$ in the boundary follows from Fefferman's Monge-Ampère algorithm in [14], applied in local coordinates to the function $\rho_{0}^{\frac{-1}{n+1}} \cdot \psi$. This amounts to solving the following problem asymptotically at $\partial X=\{\phi=0\}$ locally: find a strictly plurisubharmonic defining function $\phi$, so $\phi<0$ on $X$, and verifying

$$
J(\phi) \equiv \operatorname{det}\left(\begin{array}{cc}
\phi & \phi_{\bar{j}} \\
\phi_{i} & \phi_{i \bar{j}}
\end{array}\right)=-1 \quad \bmod O\left(\phi^{n+1}\right) .
$$

Fefferman shows that (2.8) has a solution $\phi$ which is unique mod $O\left(\phi^{n+2}\right)$. Then the volume form is uniquely defined through order $n+1$, that is, any two solutions $v_{1}, v_{2}$ of (2.7) satisfy

$$
\frac{v_{1}}{v_{2}}=1+O\left(\phi^{n+2}\right) .
$$

The transformation law in [14] relating computations in different holomorphic coordinate systems shows that the solution to equation (2.7) is globally defined near $M$, and can be extended to all of $X$ as a smooth volume form.

Following [14], p. 400, we want to improve the degree of approximation to the exact Kähler-Einstein metric by adding logarithmic terms at the boundary to $\phi$. 
Consider functions of the form

$$
\psi=\phi\left(1+A \phi^{n+1} \log (-\phi)\right),
$$

where $A$ is smooth to the boundary of $X$.

Proposition 2.1. There exists $A \in \mathcal{C}^{\infty}(\bar{X})$ such that

$$
J(\psi)=-1+O\left(\phi^{n+2} \log (-\phi)\right),
$$

and $A$ is unique mod $O(\phi)$, and is algebraically computable locally at $\partial X$.

Proof. One simply inserts (2.10) into the calculation on pp. 399-400 of [14], and arrives at the following:

$$
J(\psi)+1=-(n+3) A \phi^{n+1}+O\left(\phi^{n+2} \log (-\phi)\right),
$$

so the proposition is proved by taking

$$
A=-\frac{1+J(\phi)}{(n+3) \phi^{n+1}} .
$$

Let $g$ now be the metric

$$
g_{i \bar{j}}=\frac{\partial^{2}}{\partial z_{i} \partial \bar{z}_{j}} \log \left(\frac{1}{-\psi}\right)
$$

where $\psi$ is one of the solutions of (2.11). Equation (2.11) implies that for $g$,

$$
\text { Ric }=-(n+1) g \quad \bmod O\left(\phi^{n} \log (-\phi)\right),
$$

and more precisely,

$R i c_{i \bar{j}}+(n+1) g_{i \bar{j}}=E_{i \ell \bar{j}}^{\ell}:=E_{i \bar{j}}=\phi_{i} \phi_{\bar{j}} \phi^{n} \log (-\phi) \cdot O(1)+\phi^{n+1} \log (-\phi) \cdot O(1)$.

Note that $E_{i k \bar{\ell}}^{j}=\frac{1}{(n+1)}\left\{\delta_{i}^{j} E_{k \bar{\ell}}+\delta_{k}^{j} E_{i \bar{\ell}}\right\}$. We have the simple identity

$$
\Omega=W+R i c c i=W+E+K \text {. }
$$

Finally, define

$$
T_{j}(\Omega)=\left(\frac{i}{2 \pi}\right)^{j} \operatorname{Tr}\left(\Omega^{j}\right), j=1, \ldots, n,
$$

and the characteristic forms

$$
P_{j}(\Omega)=\sum_{k=0}^{j-2}(-1)^{k}\left(\begin{array}{l}
j \\
k
\end{array}\right) \frac{T_{j-k}(\Omega) T_{1}^{k}(\Omega)}{(n+1)^{k}}+\frac{(-1)^{j-1}(j-1) T_{1}^{j}(\Omega)}{(n+1)^{j-1}}, j=2, \ldots, n .
$$

The renormalized characteristic forms of [7] are the polynomials in the forms $P_{j}(\Omega)$. Theorem 2.1 of $[7]$ says that $P_{j}(W+K)=\left(\frac{i}{2 \pi}\right)^{j} \operatorname{Tr}\left(W^{j}\right)$. 
Proposition 2.2. For $\psi$ as in proposition 2.1, the characteristic forms $P_{j}(\Omega)$ are continuous up to the boundary of $X$, and agree with $\left(\frac{i}{2 \pi}\right)^{j} \operatorname{Tr}\left(W^{j}\right)$ there.

We need some lemmas to show this.

Lemma 2.3. For $j=1, \ldots, n$, we have an expansion

$$
T_{j}(\Omega)=\sum_{k=0}^{j} \omega^{k} b_{k},
$$

where $\omega$ is the Kähler form for $g$, and the coefficients $b_{k}$ are bounded 2j-2k forms.

Proof. This follows directly from two facts: $\Omega=\Omega-K+K=W+E+K$, where $W$ is continuous on $\bar{X}(c f .,[7]$, p. 36 and the proof of proposition 2.1 of that paper) as is $E$, by $(2.13)$; and $K \cdot(\Omega-K)=(\Omega-K) \cdot K=2 \sqrt{-1} \omega(\Omega-K)$ (proof identical to that of lemma 2.1 of $[7]$ ).

Next we estimate the difference between the trace powers of $\Omega$ and those of $W+K$.

Lemma 2.4. For $j=1, \ldots, n$, we have an error estimate

$$
\operatorname{Tr}\left(\Omega^{j}\right)-\operatorname{Tr}\left((W+K)^{j}\right)=O\left(\phi^{n+1-j} \log (-\phi)\right) .
$$

Proof. The difference of the two terms on the left hand side is expressible as a sum of terms of the form $\operatorname{Tr}\left(A_{1} \ldots A_{j}\right)$, where each of the $A_{i}$ is an $n \times n$ matrix of two forms, each one equal to one of $W, K$, or $E$, and at least one of them equal to $E$. Since $W$ is continuous on $\bar{X}$, the largest of these terms is equal to $\operatorname{Tr}\left((K)^{j-1} E\right)=(2 \sqrt{-1} \omega)^{j-1} \operatorname{Tr}(E)=(2 \sqrt{-1} \omega)^{j-1} \wedge\left\{\phi^{n} \log (-\phi) \partial \phi \wedge \bar{\partial} \phi \cdot O(1)+\right.$ $\left.O\left(\phi^{n+1} \log (-\phi)\right)\right\}$, by (2.13). The lemma follows immediately.

Proof. (of proposition 2.2) We write $P_{j}(\Omega)$ as in (2.16), and estimate $P_{j}(\Omega)-$ $P_{j}(W+K)$ term-by-term from (2.16). For example, $T_{j-k}(\Omega) T_{1}^{k}(\Omega)-T_{j-k}(W+$ $K) T_{1}^{k}(W+K)=\left\{T_{j-k}(\Omega)-T_{j-k}(W+K)\right\} T_{1}^{k}(\Omega)+T_{j-k}(W+K)\left\{T_{1}^{k}(\Omega)-\right.$ $\left.T_{1}^{k}(W+K)\right\}=O\left(\phi^{n+1-j} \log (-\phi)\right)$, by $(2.17)$ and lemma 2.4. Since $P_{j}(W+K)=$ $\left(\frac{i}{2 \pi}\right)^{j} \operatorname{Tr}\left(W^{j}\right)$ is continuous on $\bar{X}$, this proves the proposition.

Corollary 2.1. If the boundary $M$ of $X$ is spherical, then the renormalized characteristic forms of $X$, computed using any approximate $\psi$ as above, vanish at $M$.

Proof. One can choose an approximate $\psi$ which actually solves the Monge-Ampère equation exactly, and for which $W \equiv 0$. But this implies that $W$ for any approximation as above, good through log order terms, has $W=0$ at the boundary. (For $n=2$, one must use the argument of [7], proposition 2.1.) But then $P_{j}(\Omega)$ vanishes at the boundary, by proposition 2.2 . 
We have now shown that using the function $\psi$ generates approximate KählerEinstein metrics with all renormalized classes well-defined and continuous on $\bar{X}$. Of course, there are choices for $\psi$ and we would like to see how these forms depend on such a choice. Suppose we have a one parameter family of hermitian metrics which are Kähler near the boundary, $g_{t}$, smooth in $t \in[0,1]$. Let $\theta_{t}$ be the local canonical hermitian connection (1,0)-forms and $\Omega_{t}=d \theta_{t}-\theta_{t} \wedge \theta_{t}$ the corresponding curvature two forms. Recall the basic transgression formula:

$$
\operatorname{Tr}\left(\Omega_{1}^{j}\right)-\operatorname{Tr}\left(\Omega_{0}^{j}\right)=d\left\{-j \int_{0}^{1} \operatorname{Tr}\left(\left(\dot{\theta}_{t} \wedge \Omega_{t}^{j-1}\right) d t\right\} .\right.
$$

Now suppose we have two functions $\psi_{0}, \psi_{1}$ as in (2.13), where $\psi_{0}$ is of the form given in (2.10), and we allow $\psi_{1}$ to be slightly more singular, but so that

$$
\frac{\psi_{1}}{\psi_{0}}=1+B \phi^{n+1}+C \phi^{n+2} \log (-\phi),
$$

where $B \in \mathcal{C}^{\infty}(\bar{X})$ and $C$ is at least $\mathcal{C}^{2}(\bar{X}) \cap \mathcal{C}^{\infty}(X)$ on $\bar{X}$ and its third derivatives are $O(\log (-\phi))$ at the boundary and fourth derivatives $O\left(\frac{1}{\phi}\right)$. This allows the possibility of using $\psi_{1}=\psi_{K E}$, the exact Kähler-Einstein solution [9], if such exists on $X$, whose boundary behavior from [20] falls into the class described by (2.20). Then set $\psi_{t}:=(1-t) \psi_{0}+t \psi_{1}$ and set

$$
g_{t}=\partial \bar{\partial} \log \left(\frac{1}{-\psi_{t}}\right)
$$

Notice that each of $\psi_{t}$ satisfies (2.13) and (2.20). To estimate $\dot{\theta}_{t}$, we note first that

$$
\dot{\theta}_{t, i}^{j}=\frac{d}{d t}\left(g_{t}^{j \bar{k}}\right) \partial g_{t, i \bar{k}}+g_{t}^{j \bar{k}} \partial \dot{g}_{t, i \bar{k}}=-g_{t}^{j \bar{v}} \dot{g}_{t, u \bar{v}} g_{t}^{u \bar{k}} \partial g_{t, i \bar{k}}+g_{t}^{j \bar{k}} \partial \dot{g}_{t, i \bar{k}}
$$

We estimate the pieces of (2.22) as follows, starting with the obvious estimates on $\psi_{t}$. Note that for simplicity, we usually suppress explicit mention of the parameter $t$ in $\psi_{t}$, etc., in the next calculations.

$$
\begin{aligned}
\dot{\psi} & =O\left(\phi^{n+2}\right) \\
\dot{\psi}_{i} & =O\left(\phi^{n+1}\right) \psi_{i}+O\left(\phi^{n+2}\right) \\
\dot{\psi}_{\bar{k}} & =O\left(\phi^{n+1}\right) \psi_{\bar{k}}+O\left(\phi^{n+2}\right) \\
\dot{\psi}_{i \bar{k}} & =O\left(\phi^{n}\right) \psi_{i} \psi_{\bar{k}}+O\left(\phi^{n+1}\right) \psi_{i}+O\left(\phi^{n+1}\right) \psi_{\bar{k}}+O\left(\phi^{n+2}\right)
\end{aligned}
$$


Estimate the derivatives of $g$, using (2.23):

$$
\begin{aligned}
\dot{g}_{u \bar{v}} & =O\left(\phi^{n-1}\right) \psi_{u} \psi_{\bar{v}}+O\left(\phi^{n}\right) \\
\partial \dot{g}_{u \bar{v}} & =O\left(\phi^{n-2}\right) \psi_{\bar{k}}+O\left(\phi^{n-1}\right) \\
\partial g_{i \bar{k}} & =O\left(\phi^{-3}\right) \psi_{\bar{k}}+O\left(\phi^{-2}\right)
\end{aligned}
$$

Next, we draw on two estimates on $g_{t}$ from [7]: [7], equation (2.8):

$$
g^{u \bar{k}}=O(\phi)
$$

and [7], equation (2.10) ([20], equation (2.9)):

$$
g^{u \bar{k}} \psi_{\bar{k}}=O\left(\phi^{2}\right) .
$$

With these pieces in hand, the following two lemmas summarize exactly what is needed to estimate (2.19).

\section{Lemma 2.5.}

$$
\dot{\theta}_{i}^{j}=O\left(\phi^{n}\right)
$$

Proof. Using (2.25) and (2.26), we get

which taken together prove the lemma.

$$
\begin{gathered}
g^{j \bar{k}} \partial \dot{g}_{i \bar{k}}=O\left(\phi^{n}\right), \\
g^{j \bar{v}} \dot{g}_{u \bar{v}}=O\left(\phi^{n+1}\right), \\
g^{u \bar{k}} \partial g_{i \bar{k}}=O\left(\phi^{-1}\right),
\end{gathered}
$$

The second lemma is slightly more precise.

\section{Lemma 2.6.}

$$
\operatorname{Tr}(\dot{\theta})=O\left(\phi^{n}\right) \partial \phi+O\left(\phi^{n+1}\right)
$$

Proof. We compute

$$
\operatorname{Tr}(\dot{\theta})=\frac{d}{d t} \operatorname{Tr}(\theta)=\frac{d}{d t} \partial \log \left(\operatorname{det}\left(g_{i \bar{j}}\right)\right)=\frac{d}{d t} \partial \log \frac{1}{(-\psi)^{n+1}}+\frac{d}{d t} \partial \log \left(1+F \phi^{n+1}\right) .
$$

Since

and

$$
\frac{d}{d t} \partial \log \frac{1}{(-\psi)^{n+1}}=-(n+1) \frac{d}{d t} \frac{\partial \psi}{\psi}=O\left(\phi^{n}\right) \partial \phi+O\left(\phi^{n+1}\right),
$$

$$
\frac{d}{d t} \partial \log \left(1+F \phi^{n+1}\right)=\partial \frac{\dot{F} \phi^{n+1}}{1+F \phi^{n+1}}=O\left(\phi^{n}\right) \partial \phi+O\left(\phi^{n+1}\right),
$$


taken together they prove the lemma.

Proposition 2.7. Let $\psi_{i}, \Omega_{i}$ be as above, and $P(\Omega)$ a characteristic form of degree $2 k$. Then

$$
P\left(\Omega_{1}\right)-P\left(\Omega_{0}\right)=d T P,
$$

where $T P$ is a continuous form on $\bar{X}$ which vanishes along $M=\partial X$.

Proposition 2.7 has two immediate consequences.

Corollary 2.2. For $P(\Omega)$ a renormalized Chern form of degree $2 n$, the integral $\int_{X} P(\Omega)$ is independent of the approximation used to compute the approximate Kähler-Einstein metric above. In particular, if $X$ admits a complete KählerEinstein metric, these integrals agree with those introduced in [7].

Proof. This follows from proposition 2.7 and integration by parts.

Corollary 2.3. If $\partial X=M$ is a spherical CR-hypersurface, then the renormalized Chern form $P(\Omega)$ calculated in any approximate Kähler-Einstein metric vanishes at the boundary. Any two such approximations differ by a form dTP, where $T P$ vanishes on the boundary. In particular, the cohomology class $[P(\Omega)] \in$ $H^{2 i}(X, M ; \mathbb{R})$ is independent of the approximation used.

Proof. Locally at $\partial X$ we may introduce coordinates where we have a local defining function $\psi_{1}$ such that the associated metric $g_{1}$ is complex hyperbolic ( see section 3 . below). In particular, $W_{1}$ vanishes identically. Then from the argument of proposition 2.1 in [7] (if $n=2$; otherwise it is a trivial remark), any other $W=W_{0}$ also vanishes along $\partial X$. So, both $P\left(\Omega_{1}\right)$ and $P\left(\Omega_{0}\right)$ give classes in $H^{2 i}(X, M ; \mathbb{R})$, and proposition 2.7 shows that these classes are the same.

We now prove proposition 2.7 .

Proof. We start by estimating (2.19). We decompose

$$
\Omega_{t}=W_{t}+E_{t}+K_{t},
$$

where we have

$$
K_{t} \Omega_{t}=\Omega_{t} K_{t}=(2 i) \omega_{t} \Omega_{t},
$$

and similarly for $E_{t}$. We also have $E_{t}=O\left(\phi^{n} \log (-\phi)\right)$, from (2.13). Substituting (2.30) and (2.31) into the integrand of the transgression formula $\operatorname{Tr}\left(\dot{\theta}_{t} \wedge \Omega_{t}^{j-1}\right)$, we see it may be expanded into a sum

$$
\operatorname{Tr}\left(\dot{\theta}_{t} \wedge \Omega_{t}^{j-1}\right)=\sum_{k=0}^{j-1} \omega_{t}^{k} B_{k},
$$


where $B_{k}$ is a sum of terms of the form

$$
\operatorname{Tr}\left(\dot{\theta}_{t} \wedge A_{k, 1} \wedge \ldots \wedge A_{k, j-1-k}\right),
$$

and each of $A_{k, \ell}, \ell=0, \ldots, j-1$, is either $W_{t}$ or $E_{t}$. Then we have estimates:

$$
\operatorname{Tr}\left(\dot{\theta}_{t} \wedge A_{k, 1} \wedge \ldots \wedge A_{k, j-1-k}\right)=\left\{\begin{array}{l}
O\left(\phi^{n}\right) \partial \phi+O\left(\phi^{n+1}\right), k=j-1 \\
O\left(\phi^{n}\right), k \leq j-2
\end{array}\right.
$$

The case $k=j-1$ is lemma 2.6. The case $k \leq j-2$ is lemma 2.5.

Substituting (2.33) into (2.32) we get

$$
\begin{aligned}
\operatorname{Tr}\left(\dot{\theta}_{t} \wedge \Omega_{t}^{j-1}\right)= & \omega_{t}^{j-1} \wedge\left(O\left(\phi^{n}\right) \partial \phi+O\left(\phi^{n+1}\right)\right) \\
& \left.+\sum_{k \leq j-2} \omega_{t}^{k} O\left(\phi^{n}\right)\right) \\
= & O(\phi) .
\end{aligned}
$$

Integrating this from $t=0$ to $t=1$ gives the proposition in the case $P(\Omega)=$ $\operatorname{Tr}\left(\Omega^{j}\right)$.

The general $P(\Omega)$ is a sum of products $\operatorname{Tr}\left(\Omega^{j_{1}}\right) \ldots \operatorname{Tr}\left(\Omega^{j_{\ell}}\right)$, where $j_{1}+\ldots+j_{\ell} \leq$ $n$. The transgression formula for such a summand is a sum of terms like

$$
\text { constant } \times \int_{0}^{1} \operatorname{Tr}\left(\Omega_{t}^{j_{1}}\right) \ldots \operatorname{Tr}\left(\dot{\theta}_{t} \wedge \Omega^{j_{k}-1}\right) \ldots \operatorname{Tr}\left(\Omega_{t}^{j_{\ell}}\right) d t .
$$

Using (2.17) for each $\Omega_{t}$, we get that the integrand is a sum of terms

$$
O(1) \cdot \omega_{t}^{m} \wedge \operatorname{Tr}\left(\dot{\theta} \wedge \Omega_{t}^{j_{k}-1}\right),
$$

where $0 \leq m \leq \sum_{i \neq k} j_{i}$. Taken together with (2.34), we get

$$
\begin{aligned}
\omega_{t}^{m} \wedge \operatorname{Tr}\left(\dot{\theta}_{t} \wedge \Omega_{t}^{j_{k}-1}\right)= & \omega_{t}^{m+j_{k}-1} \wedge\left(O\left(\phi^{n}\right) \partial \phi+O\left(\phi^{n+1}\right)\right) \\
& \left.+\sum_{\ell \leq j_{k}-2} \omega_{t}^{m+\ell} O\left(\phi^{n}\right)\right) \\
= & O\left(\phi^{n-\left(m+j_{k}-1\right)}\right) \partial \phi+O\left(\phi^{n+1-\left(m+j_{k}\right)}\right) . \\
= & O(\phi),
\end{aligned}
$$

since $m+j_{k}<n+1$. Integrating from 0 to 1 proves proposition 2.7. 


\section{Development Mappings and Monodromy.}

In this section we draw some consequences of the development map technique of [5]. Since our boundary $M$ is spherical, there is a covering $\left\{U_{\alpha}\right\}$ of $M$, and CR diffeomorphisms $\phi_{\alpha}: U_{\alpha} \rightarrow \partial \mathbb{B}^{n}$. These are related on overlaps by the CR maps

$$
\phi_{\alpha, \beta}:=\phi_{\alpha} \circ \phi_{\beta}^{-1}: \phi_{\beta}\left(U_{\alpha} \cap U_{\beta}\right) \rightarrow \phi_{\alpha}\left(U_{\alpha} \cap U_{\beta}\right) \subset \partial \mathbb{B}^{n},
$$

where the $\phi_{\alpha, \beta}$ are global transformations in $\operatorname{PSU}(n, 1)$, the biholomorphic automorphism group of $\mathbb{B}^{n}$. This implies that the coordinate charts $\phi_{\alpha}$ can be analytically continued along any continuous curve $\gamma$ lying in $M$, that is, they define a multivalued CR-equivalence on all of $M$. By Levi extension, the CR mappings $\phi_{\alpha}$ extend to holomorphic mappings also denoted $\phi_{\alpha}$, defined and locally biholomorphic on relatively open sets $\widehat{U}_{\alpha} \subset X$, mapping to the (closed) unit ball $\overline{\mathbb{B}}^{n}$. Since the overlaps are given by the extensions of $\phi_{\alpha, \beta}$ to $\widehat{U}_{\alpha} \cap \widehat{U}_{\beta}$, these local extensions define a multivalued holomorphic mapping, denoted $D$, from a neighborhood of the boundary $M$ of $X$ into the unit ball $\mathbb{B}^{n}$. This mapping is unique up to composition with a global element $g \in P S U(n, 1)$, and is called the development map.

By the multivalued Hartogs theorem of [5], the multivalued development map $D$ may be analytically continued along any continuous path $c(t)$ lying in $X \backslash E$, where $E \subset X$ is the maximal compact subvariety of $X$. Fixing a basepoint $z_{0} \in X \backslash E$, and a determination of $D$ near $z_{0}$, the multivaluedness of $D$ gives a monodromy representation

$$
\rho: \pi_{1}\left(X \backslash E, z_{0}\right) \rightarrow P S U(n, 1),
$$

which is uniquely determined up to (global) conjugation in $P S U(n, 1)$. Since the monodromy of $D$ lies in $P S U(n, 1)$, there is a well-defined Kähler metric $D^{*} d s_{C H}^{2}$ pulled back from $\mathbb{B}^{n}$ by the development map. Here $d s_{C H}^{2}$ is the complex hyperbolic (Bergman) metric on $\mathbb{B}^{n}$ for which $P S U(n, 1)$ acts as isometries. This metric has constant holomorphic sectional curvature -1 . This pullback metric is locally near the boundary of the form for which we can define renormalized Chern forms, and indeed, by 2.7, such forms will vanish identically wherever this metric is defined, i.e., on $X \backslash E$. Furthermore, given any open neighborhood $\mathcal{V}$ of $E$, we may find a Hermitian metric $h$ on the holomorphic tangent bundle of $T X$ which agrees with the Kähler metric $D^{*} d s_{C H}^{2}$ in an open neighborhood of $X \backslash \mathcal{V}$. The forms $\tilde{c}_{j}, j=2, \ldots, n$, computed in $h$ vanish on this same set, and so are compactly supported in $\mathcal{V}$. By the usual transgression formula, as used in propositions 2.2 and 2.7 , the classes of the forms $P\left(\tilde{c}_{2}, \ldots, \tilde{c}_{n}\right)$ in $H^{*}(X, M ; \mathbb{R})$ are independent of the choice of such an $h$, where $P$ is any polynomial with rational coefficients as in Theorem 1.1.

Our first understanding of the principal monodromies comes from the generalized Schwarz lemma, see, for example, [17], [28]. Fix a point $z \in E^{*}$, the smooth 
locus of $E$. If $\operatorname{cod}_{z}(E)>1$, then $D$ extends holomorphically across $E$, and the codimension assumption means that $D$ is also a local diffeomorphism across $E$ in a neighborhood of $z$. This extends the locally complex hyperbolic metric $D^{*} d s_{c h}^{2}$ to a neighborhood of $z$, so we will assume that $\operatorname{cod}_{z}(E)=1$. In this case, let $\Gamma_{\mathbb{C}}$ be a complex disk in $X$ which is transverse to $E$ and such that the origin of $\Gamma_{\mathbb{C}} \cap E=\left\{z_{0}\right\}$, where $z_{0}$ is a smooth point of $E$.

In local coordinates $\left(z_{1}, \ldots, z_{n-1}, \zeta\right)$ at $0=z_{0} \in X$, so that $E_{j}$ given by $\zeta=0$, by the Schwarz lemma we have

$$
D^{*} d s_{C H}^{2} \leq C\left\{\sum_{i=1}^{n-1}\left|d z_{i}\right|^{2}+\frac{|d \zeta|^{2}}{|\zeta|^{2} \log ^{2}\left(|\zeta|^{2}\right)}\right\} .
$$

Let $c(r)=(0, \ldots, 0, r), r \in(0, \epsilon)$, be a radial curve. There is a single-valued development map $D$ along $c$. The boundedness of $\mathbb{B}^{n}$ implies that there exist a sequence $r_{i} \rightarrow 0$ in $(0, \epsilon)$, and a point $w_{0} \in \overline{\mathbb{B}^{n}}$ so that $\lim _{r_{i} \rightarrow 0} D\left(c\left(r_{i}\right)\right)=w_{0}$.

The length of the circle $C_{r}=\left\{|\zeta|=r, z_{1}=\ldots=z_{n-1}=0\right\}$ in the Poincaré metric $\frac{|d \zeta|^{2}}{|\zeta|^{2} \log ^{2}\left(|\zeta|^{2}\right)}$ is bounded by $\frac{C}{\log \frac{1}{r}}$. If we analytically continue $D$ once counterclockwise along $C_{r}$, starting at $c(r)$, we come to $\rho\left(\gamma_{j}\right) \cdot D(c(r))$. Therefore, taking complex hyperbolic distances in $\mathbb{B}^{n}$, we see that

$$
d_{C H}\left(D(c(r)), \rho\left(\gamma_{j}\right) \cdot D(c(r))\right) \leq \frac{C}{\log \frac{1}{r}} .
$$

Since the Euclidean distance in $\mathbb{B}^{n}$ is less than the complex hyperbolic distance, taking $\lim _{r_{i} \rightarrow 0}$ of this last equation, we conclude that $\rho\left(\gamma_{j}\right)\left(w_{0}\right)=w_{0}$.

Proposition 3.1. The principal monodromy $\gamma_{j}$ has $\rho\left(\gamma_{j}\right) \in P S U(n, 1)$ which is either elliptic or parabolic.

Proof. If $w_{0}$ is in $\mathbb{B}^{n}$, then $\rho\left(\gamma_{j}\right)$ is elliptic. If $w_{0} \in \partial \mathbb{B}^{n}$, then by applying a Cayley transformation, we may without loss of generality replace $\mathbb{B}^{n}$ by its Siegel domain representation $\mathcal{U}_{n}=\left\{(z, w) \in \mathbb{C}^{n-1} \times\left.\mathbb{C}|\operatorname{Im}(w)-| z\right|^{2}>0\right\}$, and that $w_{0}$ gets sent to the point at infinity. In this setting, $\rho\left(\gamma_{j}\right)$ is represented as follows:

$$
\rho\left(\gamma_{j}\right)(z, w)=\left(\lambda \cdot A(z+a), \lambda^{2} \cdot(w+t+2 \sqrt{-1}(z, a)+\sqrt{-1}(a, a))\right),
$$

where $0<\lambda \in \mathbb{R}, A \in U(n-1), a \in \mathbb{C}^{n-1}$ and $t \in \mathbb{R}$, and the claim of the proposition is that $\lambda=1$.

Let $H=H(z, w),(z, w) \in \mathcal{U}_{n}$ denote the height function $H(z, w)=\operatorname{Im}(w)-$ $|z|^{2}$. Then the complex hyperbolic metric on $\mathcal{U}_{n}$ has Kähler form $\omega_{C H}=\frac{\sqrt{-1}}{2} \partial \bar{\partial}$ $\log \frac{1}{H}$, and so we have the relation between non-negative hermitian differential forms:

$$
d s_{C H}^{2} \geq \frac{\partial H}{H} \otimes \frac{\bar{\partial} H}{H} .
$$


Hence, for any real curve $u(t) \in \mathcal{U}_{n}, t \in[a, b]$, we have

$$
\left|u^{\prime}(t)\right|_{C H}^{2} \geq\left|<\frac{\partial H}{H}, u^{\prime}(t)>\right|^{2}=\frac{1}{4} \cdot\left|<\frac{d H}{H}, u^{\prime}(t)>\right|^{2},
$$

and hence,

$$
\operatorname{length}_{C H}(u) \geq \frac{1}{2} \cdot\left|\log \frac{H(u(a))}{H(u(b))}\right| .
$$

Applying this to the case where $u(t)$ is the analytic continuation of $D$ along $C_{r}, r>0$, we find

$$
\operatorname{dist}_{C H}\left(D(c(r)), \rho\left(\gamma_{j}\right) \cdot(D(c(r)))\right) \geq \frac{1}{2} \cdot\left|\log \frac{H\left(\rho\left(\gamma_{j}\right) \cdot(D(c(r)))\right)}{H(D(c(r)))}\right|=|\log \lambda| .
$$

Since $\lim _{r \rightarrow o} \operatorname{dist}_{C H}\left(D(c(r)), \rho\left(\gamma_{j}\right) \cdot(D(c(r)))=0\right.$, we conclude that $\lambda=1$.

\section{Exponents of the Principal Monodromies}

Let $A d_{\mathbb{C}}: P S U(n, 1) \rightarrow G L(\mathfrak{s u}(n, 1) \otimes \mathbb{C})$ be the complexified adjoint representation, and denote by $\rho_{\mathbb{C}}$ the representation $A d_{\mathbb{C}} \circ \rho: \pi_{1}\left(X, z_{0}\right) \rightarrow G L(\mathfrak{s u}(n, 1) \otimes$ $\mathbb{C})$. Recall that for every (smooth) irreducible component $E_{j}$ of the maximal compact subvariety $E$ of $X$, the principal monodromy of $E_{j}$ is the element in the fundamental group $\pi_{1}\left(X, z_{o}\right)$ represented by a loop $\gamma_{j}$ going once counterclockwise around the divisor $E_{j}$, e.g., the curve $C_{r}$ in the previous section. The conjugacy class of $\gamma_{j} \in \pi_{1}\left(X, z_{0}\right)$ is uniquely determined. The aim of the present section is to prove the following lemma.

Lemma 4.1. All eigenvalues of $\rho_{\mathbb{C}}\left(\gamma_{j}\right)$ are roots of unity, for every irreducible divisor $E_{j}$ of $E$.

Before proving 4.1 we make some reductions.

Lemma 4.2. In lemma 4.1 we may assume that a neighborhood of $E \subset X$ is biholomorphic to an open neighborhood of a divisor $E^{\prime}$ in a projective algebraic manifold $X^{\prime}$. Further, we may assume that every irreducible component $E_{k}^{\prime}$ of $E^{\prime}$ is non-singularly embedded in $X^{\prime}$, and that $E^{\prime}$ has normal crossings.

Proof. Let $E^{[j]}$ denote the connected components of $E$. Then each $E^{[j]}$ may be contracted to an isolated normal singular point $x^{[j]}$ in a complex variety $\check{X}$. By a theorem of $\mathrm{M}$. Artin, the space $\check{X}$ is locally biholomorphic to a projective algebraic variety $\check{Y}^{[j]}$ at $x^{[j]}$. By resolution of singularities, there is a projective manifold $\widehat{Y}$ and a proper map $\widehat{\pi}: \widehat{Y} \rightarrow \check{Y}$ such that $\widehat{\pi}$ is biregular over $\check{Y} \backslash\left\{x^{[j]}\right\}$, and $\widehat{\pi}^{-1}\left(x^{[j]}\right)=\widehat{E}^{[j]}$ is a smooth divisor with normal crossings, all of whose components are non-singularly embedded in $\widehat{Y}$. Furthermore, we may assume 
that there is a neighborhood $\widehat{\mathcal{V}}$ of $\widehat{E}=\cup \widehat{E}^{[j]}$ and a mapping $\pi: \widehat{\mathcal{V}} \rightarrow \mathcal{V} \subset X$, where $\mathcal{V}$ is a neighborhood of $E$ in $X$. This mapping is biregular off $E$, and $\pi^{-1}(E)=\widehat{E} \subset \widehat{Y}$. Note that for every irreducible divisor $E_{i}$ in $E$, the proper transform of $\left(E_{i}\right)$, say $\widehat{E}_{i}$, is among the (smooth) irreducible divisors of $\widehat{E}$, and that if $\widehat{\gamma}_{i}$ denotes a loop passing once around the divisor $\widehat{E}_{i}$, then $\pi\left(\widehat{\gamma}_{i}\right)=\gamma_{i}$ is a loop passing once positively around $E_{i}$. Thus, to prove 4.1 it suffices to consider the representation, still denoted $\rho_{\mathbb{C}}=A d \circ \rho: \pi_{1}\left(\widehat{\mathcal{V}} \backslash \widehat{E}, z_{0}\right) \rightarrow P S U(n, 1)$, and to prove that all eigenvalues of $\rho_{\mathbb{C}}\left(\widehat{\gamma}_{k}\right)$ are roots of unity, for all $\widehat{\gamma}_{k}$, principal monodromy around an irreducible divisor of $\widehat{E}$.

Henceforth, we will assume the properties of lemma 4.2 but keep the original notation $X, E$, etc. Next, we reduce $X$ to a surface which still carries the principal monodromy loops. Since $\widehat{Y}$ in the proof of 4.2 is projective, we may intersect it with generic hypersurface sections in some projective embedding so that we may construct a non-singular surface $S \subset X$ such that $S \cap E=C=\cup_{j} C_{j}$, where each $C_{j}=S \cap E_{j}$ is a smooth curve in $S$, where $E_{j}$ runs over the irreducible divisors of $E$, and every pair $C_{i}, C_{j}$ intersect transversally. By restricting to a neighborhood of $C$ in $S$, and by blowing up a few points of intersection of $C_{i}, C_{j}$ if necessary, we may assume that we have a surface $S$ which is a deformation retraction of the curve $C$ and that the irreducible components $C_{i}, C_{j}$ of $C$ either do not intersect, or if they do, they intersect transversally in just one point. In this case, every principal monodromy loop about some divisor $E_{j}$ in $X$ is in the image of a loop about one of the curves $C_{i}$. Thus, we are reduced to the following situation:

We have a surface $S$, a deformation retraction to a curve $C \subset S$, and $C=\cup_{i} C_{i}$, where the irreducible curves $C_{i}$ are smooth and intersect each other transversally at no more than one point per pair of curves. We have a representation, still denoted $\rho_{\mathbb{C}}$, coming from our original monodromy representation,

$\rho_{\mathbb{C}}=A d_{\mathbb{C}} \circ \rho \circ \iota_{\#}: \pi_{1}\left(S \backslash C, z_{0}\right) \rightarrow \pi_{1}\left(X \backslash E, z_{0}\right) \rightarrow P S U(n, 1) \rightarrow G L(\mathfrak{s u}(n, 1) \otimes \mathbb{C})$,

where $\iota: S \backslash C \rightarrow X \backslash E$ is the inclusion, and $\iota_{\#}$ is the induced map of fundamental groups. It remains to show that all eigenvalues of $\rho_{\mathbb{C}}\left(\gamma_{i}\right)$ are roots of unity, where $\gamma_{i}$ runs over all the principal monodromies of the components $C_{i}$.

We may take $S$ above to be a tubular neighborhood of $C$ of the form given in [22], which is constructed from the normal disk bundles of the $C_{i} \subset S$ plumbed together at the various intersection points of the components. We recall Mumford's description of the fundamental group $\pi_{1}\left(S \backslash C, z_{0}\right)$ in terms of generators and relations in this situation. Concentrate first on an irreducible component $C_{i}$ of $C$. Let $N_{i}$ be the holomorphic normal bundle of $C_{i} \subset S$ and $B_{i} \subset N_{i}$ the unit disk bundle. Let $\pi_{i}: B_{i} \rightarrow C_{i}$ be the projection. Let $x_{i, j}=C_{i} \cap C_{j}$, if this intersection is non-empty. Let $g_{i}$ be the genus of $C_{i}$. The fundamental group 
$\pi_{1}\left(C_{i} \backslash\left\{x_{i, j}\right\}, z_{i}\right)$ is generated by curves $\alpha_{k}^{i}, \beta_{k}^{i}, k=1, \ldots, g_{i}$, and $c_{i, j}$, where the $\alpha_{k}$ and $\beta_{k}$ are a standard generating set for the fundamental group of $C_{i}$, and for each intersection point $x_{i, j}, c_{i, j}$ is given by a loop passing once counter-clockwise about $x_{i, j}$. The group $\pi_{1}\left(C_{i} \backslash\left\{x_{i, j}\right\}, z_{i}\right)$ is generated by these elements subject to the single non-trivial relation

$$
1=\prod_{k=1}^{g_{i}}\left[\alpha_{k}^{i}, \beta_{k}^{i}\right] \cdot \prod_{j} c_{i, j}^{-1}
$$

where $\left[\alpha_{k}^{i}, \beta_{k}^{i}\right]=\alpha_{k}^{i} \beta_{k}^{i} \alpha_{k}^{i-1} \beta_{k}^{i-1}$ is the commutator.

Next let $W_{i}=\pi_{i}^{-1}\left(C_{i} \backslash\left\{x_{i, j}\right\}\right)$ minus the zero section. The fundamental group of $W_{i}$ is represented in terms of generators $\bar{\alpha}_{k}^{i}, \bar{\beta}_{k}^{i}, k=1, \ldots, g_{i}, \bar{c}_{i, j}$, where $\pi_{i}\left(\bar{\alpha}_{k}^{i}\right)=\alpha_{k}^{i}$, etc., and one new generator $\gamma_{i}$ going once around 0 in the fiber of $B_{i}$, which is the principal monodromy corresponding to the component $C_{i}$. The fundamental group $\pi_{1}\left(W_{i}, \bar{z}_{i}\right)$ is generated by these elements. They verify the relation

$$
\gamma_{i}^{d_{i}}=\prod_{k=1}^{g_{i}}\left[\bar{\alpha}_{k}^{i}, \bar{\beta}_{k}^{i}\right] \cdot \prod_{j} \bar{c}_{i, j}^{-1},
$$

where $d_{i}$ is the self-intersection number $C_{i} \cdot C_{i}$ of $C$ in $S$ (the degree of $N_{i}$ ). Furthermore, $\gamma_{i}$ is central in $\pi_{1}\left(W_{i}, \bar{z}_{i}\right)$. In $S, C_{i}$ and $C_{j}$ are joined at the point $x_{i, j}=x_{j, i}=C_{i} \cap C_{j} . W_{i}$ and $W_{j}$ are plumbed together in such a way that $\gamma_{i}$ is identified with $\bar{c}_{j, i}$ and $\gamma_{j}$ with $\bar{c}_{i, j}$. Note that we will eventually have to be careful about the question of basepoints for fundamental groups, but that the statement of lemma 4.1 only involves the conjugacy classes of the $\gamma_{i}$ 's, which simplifies things considerably.

Proof. (of Lemma 4.1) We would like, in some sense, to abelianize the representation $\rho_{\mathbb{C}}$. This is possible if we can decompose the representation according to eigenspaces, which is almost possible. To carry this out, define $V_{\ell}^{i} \subset$ $\mathfrak{s u}(n, 1) \otimes \mathbb{C}$ to be the $\lambda_{\ell}^{i}$ generalized eigenspace for $\rho_{\mathbb{C}}\left(\gamma_{i}\right)$, i.e., the kernel of $\left(\rho_{\mathbb{C}}\left(\gamma_{i}\right)-\lambda_{\ell}^{i} I\right)^{N}, N>>0$. Since $\gamma_{i}$ is central in $\pi_{1}\left(W_{i}, \bar{z}_{i}\right)$, the subspace $V_{\ell}^{i}$ is left stable by $\rho_{\mathbb{C}}\left(\bar{\alpha}_{k}^{i}\right), \rho_{\mathbb{C}}\left(\bar{\beta}_{k}^{i}\right), \rho_{\mathbb{C}}\left(\bar{c}_{i, j}\right)$, for all $k, j$. We may restrict the relation (4.2) above to $V_{\ell}^{i}$,and get

$$
\left(\left.\rho_{\mathbb{C}}\left(\gamma_{i}\right)\right|_{V_{\ell}^{i}}\right)^{d_{i}}=\left.\prod_{k=1}^{g_{i}}\left[\left.\rho_{\mathbb{C}}\left(\bar{\alpha}_{k}^{i}\right)\right|_{V_{\ell}^{i}},\left.\rho_{\mathbb{C}}\left(\bar{\beta}_{k}^{i}\right)\right|_{V_{\ell}^{i}}\right] \prod_{j} \rho_{\mathbb{C}}\left(\bar{c}_{i, j}\right)^{-1}\right|_{V_{\ell}^{i}}
$$

Taking determinants of this relationship gives us equations

$$
\left(\lambda_{\ell}^{i}\right)^{d_{i} \operatorname{dim} V_{\ell}^{i}}=\prod_{j} \operatorname{det}\left(\left.\rho_{\mathbb{C}}\left(\bar{c}_{i, j}\right)^{-1}\right|_{V_{\ell}^{i}}\right),
$$


one for each $\ell$, where $\ell$ runs over the distinct eigenvalues of $\rho_{\mathbb{C}}\left(\gamma_{i}\right)$. This gives us as many equations as there are unknowns in our problem, namely, the number of distinct eigenvalues of the $\rho_{\mathbb{C}}\left(\gamma_{i}\right)$ 's. Unfortunately, we cannot yet describe this system clearly enough to analyze it. This is due to the role played by the choice of basepoints for the fundamental groups involved. To see this, consider a simpler case where we have a representation of $\pi_{1}\left(S \backslash C, \bar{z}_{0}\right)$ in $G L(2, \mathbb{C})$, and $C$ has three distinct components which form a triangle in $S$. Assume we have distinct eigenvalues $\lambda_{\ell}^{i}, i=1,2,3$, and $\ell=1,2$. Now suppose we start making equations for the eigenvalues from the first component: we would get two equations:

$$
\begin{aligned}
& \lambda_{1}^{1^{d_{1}}} \cdot \lambda_{1}^{2} \cdot \lambda_{1}^{3}=1, \\
& \lambda_{2}^{1^{d_{1}}} \cdot \lambda_{2}^{2} \cdot \lambda_{2}^{3}=1 .
\end{aligned}
$$

Unfortunately, when one writes down analogous equations generated from the component $C_{2}$ and its principal monodromy element $\gamma_{2}$, the names of the eigenvalues of $\gamma_{1}$ and $\gamma_{3}$ may not be consistent. We will see below that in order to analyze the system of equations, it is important that the eigenvalues be labeled consistently, so that the next set of equations is

$$
\begin{aligned}
& \lambda_{1}^{2^{d_{2}}} \cdot \lambda_{1}^{1} \cdot \lambda_{1}^{3}=1, \\
& \lambda_{2}^{2}{ }^{d_{2}} \cdot \lambda_{2}^{1} \cdot \lambda_{2}^{3}=1,
\end{aligned}
$$

and not, for example,

$$
\begin{aligned}
& \lambda_{1}^{2^{d_{2}}} \cdot \lambda_{1}^{1} \cdot \lambda_{2}^{3}=1, \\
& \lambda_{2}^{2 d_{2}} \cdot \lambda_{2}^{1} \cdot \lambda_{1}^{3}=1 .
\end{aligned}
$$

To this end, we consider the intersection graph $K$ of the array of curves $C=\cup_{i} C_{i}$. Recall that one introduces a line segment $L_{i}$ for each $C_{i}$, and $L_{i}$ and $L_{j}$ are joined at one point if $L_{i} \cap L_{j} \neq \phi$. This point of intersection is distinct for distinct pairs $L_{i}, L_{j}$. We would like to construct next a finite covering $\widehat{K}$ of $K$. Over each $L_{i}$ introduce $m:=n^{2}+2 n=\operatorname{dim}_{\mathbb{C}} \mathfrak{s u}(n, 1) \otimes \mathbb{C}$ distinct line segments $\tilde{L}_{1}^{i}, \ldots, \tilde{L}_{m}^{i}$. Associate with each $\tilde{L}_{k}^{i}$ an eigenvalue $\lambda_{k}^{i}$ of $\rho_{\mathbb{C}}\left(\gamma_{i}\right)$. Do this in such a way that one has exactly $m_{\ell}=$ multiplicity $\lambda_{\ell}^{i}$ of the $\lambda_{k}^{i}$ equal to $\lambda_{\ell}^{i}$, for all $\ell$, where $\lambda_{\ell}^{i}$ runs, as before, over the distinct eigenvalues of $\rho_{\mathbb{C}}\left(\gamma_{i}\right)$. Care must be taken in how we join the various $\tilde{L}_{k}^{i}, \tilde{L}_{k^{\prime}}^{i^{\prime}}$ together over $K$. Letting $V\left(\lambda, \rho_{\mathbb{C}}\left(\gamma_{i}\right)\right) \subset \mathfrak{s u}(n, 1) \otimes \mathbb{C}$ denote the generalized eigenspace for $\rho_{\mathbb{C}}\left(\gamma_{i}\right)$ with eigenvalue $\lambda$, and similarly for $V\left(\lambda, \rho_{\mathbb{C}}\left(\bar{c}_{i, i^{\prime}}\right)\right)$. If $L_{i} \cap L_{i^{\prime}} \neq \phi$ in $K$, then join arbitrarily $m\left(i, k ; i^{\prime}, k^{\prime}\right):=\operatorname{dim} V\left(\lambda_{k}^{i}, \rho_{\mathbb{C}}\left(\gamma_{i}\right)\right) \cap V\left(\lambda_{k^{\prime}}^{i^{\prime}}, \rho_{\mathbb{C}}\left(\bar{c}_{i, i^{\prime}}\right)\right)$ of the $\tilde{L}_{k}^{i}$ with the $\tilde{L}_{k^{\prime}}^{i^{\prime}}$

There is a natural covering map $p: \widehat{K} \rightarrow K$ of degree $m$. There is another, connected covering of $q: K^{\prime} \rightarrow K$ of degree $\leq m$ ! such that the induced covering 
$\widehat{K}^{\prime}=q^{*} \widehat{K}$ is trivial, i.e., is equivalent to $K^{\prime} \times\{1, \ldots, m\}$. We next use $K^{\prime}$ to induce a covering of $S$. We already have a retraction $r: S \rightarrow C$. On the other hand, it is clear that we have a surjection $s: C \rightarrow K$, and together these induce a surjection $s_{\#} \circ r_{\#}: \pi_{1}\left(S, z_{0}\right) \rightarrow \pi_{1}\left(K, x_{0}\right)$. The covering $K^{\prime} \rightarrow K$ corresponds to a finite index subgroup $\pi_{K^{\prime}} \subset \pi_{1}\left(K, x_{0}\right)$ and so we induce the finite index subgroup $\pi_{S^{\prime}} \subset \pi_{1}\left(S, z_{0}\right)$, which corresponds in turn to a finite covering $q_{S^{\prime}}: S^{\prime} \rightarrow S$. By construction, $C^{\prime}=q_{S^{\prime}}^{-1}(C)$ is a connected curve whose irreducible components are embedded smooth curves, and which intersect each other in at most one point. Consider the principal monodromies $\gamma_{i^{\prime}}^{\prime}$ around the components $C_{i}^{\prime}$ of $C^{\prime}$, and their representation, still denoted $\rho_{\mathbb{C}}$, via $\pi_{1}\left(S^{\prime}, z_{0}^{\prime}\right) \rightarrow \pi_{1}\left(S, z_{0}\right) \rightarrow G L(\mathfrak{s u}(n, 1) \otimes$ $\mathbb{C}) . q_{S^{\prime}}$ sends $C_{i^{\prime}}^{\prime}$ to some component $C_{i}$ of $C$, and there exists a positive integer $\nu\left(i, i^{\prime}\right)$ such that $\rho_{\mathbb{C}}\left(\gamma_{i^{\prime}}^{\prime}\right)=\left(\rho_{\mathbb{C}}\left(\gamma_{i}\right)\right)^{\nu\left(i, i^{\prime}\right)}$. Hence, to prove lemma 4.1 it suffices to show that all eigenvalues of $\rho_{\mathbb{C}}\left(\gamma_{i^{\prime}}^{\prime}\right)$ are roots of unity, for all $i^{\prime}$.

We will now drop the primes from our notation, but assume instead that for the eigenvalues (listed with multiplicity) $\lambda_{k}^{i}$ of each of the $\rho_{\mathbb{C}}\left(\gamma_{i}\right)$ we have an ordering, say $k=1, \ldots, m$, which is independent of passing around closed loops of components $C_{j}$ (i.e., well-defined after passing around closed loops in $K$ ).

Let us next revisit the equations on the $\lambda_{k}^{i}$ described in (4.4). First of all, we fix some notation. Let $s=$ the number of components $C_{i}$ of $C$. For $k, k^{\prime}=1, \ldots, m$, let $(i, k) \sim\left(i, k^{\prime}\right)$ if $\lambda_{k}^{i}=\lambda_{k^{\prime}}^{i}$. Let $u, v$ run over the set of $\sim$ equivalence classes $[i, k], i=1, \ldots, s, k=1, \ldots, m$, and let $N$ denote the number of distinct $\sim$ equivalence classes. Let $\lambda(u)=\lambda_{k}^{i}$, for any $(i, k) \in u$.

Next, let us note that, since the $\rho\left(\gamma_{i}\right)$ are either elliptic or parabolic in $\operatorname{PSU}(n, 1)$, we have that $\left|\lambda_{k}^{i}\right|=1$, for all $i, k$. Hence we can write $\lambda_{k}^{i}=e^{2 \pi \sqrt{-1}} a_{k}^{i}$, where $a_{k}^{i} \in \mathbb{R}$, and they are well-defined $\bmod \mathbb{Z}$. As above, we set $a(u)=a_{k}^{i}$, for any $(i, k) \in u$.This means that, taking $\frac{1}{2 \pi \sqrt{-1}} \log$, equation (4.4) is equivalent to the following, for every class $u$ :

$$
\sum_{(i, k) \in u}\left\{d_{i} a_{k}^{i}+\sum_{\left\{i^{\prime} \mid C_{i} \cap C_{i^{\prime}} \neq \phi\right\}} a_{k}^{i^{\prime}}\right\} \equiv 0 \quad \bmod \mathbb{Z} .
$$

Let $\left(C_{i, j}\right)=\left(C_{i} \cdot C_{j}\right)$ be the (symmetric) intersection matrix of the curves $C_{i}, C_{j}$ in $S$. Notice that (4.5) can be rewritten

$$
\sum_{\{k \mid(i, k) \in u\}} \sum_{j=1}^{s} C_{i, j} a_{k}^{j} \equiv 0 \bmod \mathbb{Z} .
$$

Concerning the matrix $\left(C_{i, j}\right)$, we note that the curve $C$ may be contracted to an isolated normal surface singularity, since, e.g., it has a strongly pseudoconvex neighborhood in $S$. By a well-known theorem of Mumford [22], the matrix $\left(C_{i, j}\right)$ is negative definite. 
Returning to our system of equations for the $a(u)$, we can write them in terms of an $N \times N$ matrix $A=\left(A_{u, v}\right)$ with integer entries. For each $t=1, \ldots, m$, let $I_{t}=\left\{u \mid \lambda(u)=\lambda_{t}^{i}\right.$, for some $\left.1 \leq i \leq s\right\}$, and define the subspace $\mathbb{R}_{[t]}^{s}=$ $\left\{\sum_{i \in I_{t}} c_{i} e_{i}, c_{i} \in \mathbb{R}\right\} \subset \mathbb{R}^{N}$, where $e_{i}$ is the standard basis of $\mathbb{R}^{N}$. Let $\Pi_{t}$ : $\mathbb{R}^{N} \rightarrow \mathbb{R}_{[t]}^{s}$ be the orthogonal projection sending $\sum_{i=1}^{s} c_{i} e_{i}$ to $\sum_{i \in I_{t}} c_{i} e_{i}$. Define the symmetric $N \times N$ matrix $A^{[t]}$ by

$$
A_{u, v}^{[t]}= \begin{cases}C_{i} \cdot C_{i^{\prime}} & \text { if }[i, t]=u,\left[i^{\prime}, t\right]=v, \\ 0 & \text { else. }\end{cases}
$$

By Mumford's theorem, for any $w \in \mathbb{R}^{N}, \sum_{u, v} A_{u, v}^{[t]} w_{u} w_{v} \leq 0$, with strict inequality if $\Pi_{t} w \neq 0$ in $\mathbb{R}_{[t]}^{s}$.

Note that now the matrix $A$ of our system of equations may be written

$$
A=\sum_{t=1}^{m} A^{[t]},
$$

so that $A$ is negative semi-definite, and in fact, for any vector $w \in \mathbb{R}^{N}\left(w^{\tau}\right.$ denotes the transpose), we have:

$$
w^{\tau} A w=\sum_{t} w^{\tau} A^{[t]} w=\sum_{t}\left(\Pi_{t} w\right)^{\tau} A^{[t]}\left(\Pi_{t} w\right) \leq 0
$$

where equality holds if and only if $\Pi_{t} w=0$, for all $t=1, \ldots, m$, which is if and only if $w=0$. Hence, the matrix $A$ is invertible. Since $A$ has integer entries, the equations $\sum_{v} A_{u, v} a(v) \equiv 0 \bmod \mathbb{Z}$, for all $u$, implies that $a(u) \in \mathbb{Q}$, for all $u$. Therefore, every $\lambda(u)=e^{2 \pi \sqrt{-1} a(u)}$ is a root of unity, proving lemma 4.1 .

\section{Some Reductions}

In this section we exploit the main lemma 4.1 from the previous section. We first consider the group $\Gamma=\rho_{\mathbb{C}}\left(\pi_{1}\left(X \backslash E, z_{0}\right)\right) \subset G L(\mathfrak{s u}(n, 1) \otimes \mathbb{C})$. This is a finitely generated group, and by a theorem of A. Selberg ([26], lemma 8, which the authors first learned from Borel's proposition 2.3 of [3]), there is a finite index normal subgroup $\Gamma^{\prime} \subset \Gamma$ such that for every element $g \in \Gamma^{\prime}$, the only root of unity which is an eigenvalue of $g$ is 1 . Let us consider $\rho_{\mathbb{C}}^{-1}\left(\Gamma^{\prime}\right) \subset \pi_{1}\left(X \backslash E, z_{0}\right)$, a normal subgroup of finite index, which corresponds to a finite Galois covering $p: X^{\prime} \rightarrow X \backslash E$. By resolution of singularities and Riemann's existence theorem, we can assume that this extends to a proper holomorphic map $p: \widehat{X} \rightarrow X$, where $\widehat{X}$ is a complex manifold with $\widehat{E}:=p^{-1}(E)$ a divisor with normal crossings and smoothly embedded irreducible components, and for which $p: \widehat{X} \backslash \widehat{E} \rightarrow X \backslash E$ 
agrees with our original Galois covering. By construction, $\rho_{\mathbb{C}}\left(\pi_{1}\left(\widehat{X} \backslash \widehat{E}, z_{0}\right)\right)=\Gamma^{\prime}$. Note also that lemma 4.1 applies to the principal monodromies $\widehat{\gamma}_{i}$ around the components $\widehat{E}_{i}$ of $\widehat{E}$, and hence these all have all eigenvalues equal to 1 .

Next, let us pull $T X$ back to $\widehat{X}$ by $p$. By the projection formula, $\left[\tilde{c}_{j}(X)\right] \in$ $H^{2 i}(X, M ; \mathbb{Q})$ if and only if $p^{*}\left(\left[\tilde{c}_{i}\right]\right) \in H^{*}(\widehat{X}, \widehat{M} ; \mathbb{Q})$, where $\widehat{M}=\partial \widehat{X}=p^{-1}(M)$. On the other hand, we have the differential $d p_{*}: T \widehat{X} \rightarrow p^{*} T X$, which is an isomorphism off $\widehat{E}$. We can construct a hermitian metric $\widehat{h}$ on $T \widehat{X}$ which agrees with that on $p^{*} T X$ via $d p_{*}$ outside any neighborhood of $\widehat{E}$, and $\left[\tilde{c}_{i}(\widehat{X})\right]-\left[p^{*} \tilde{c}_{i}(X)\right] \in$ $H^{2 i}(\widehat{X}, \widehat{M} ; \mathbb{Q})$. Putting these two observations together, we conclude:

Proposition 5.1. $\left[\tilde{c}_{i}(X)\right] \in H^{2 i}(X, M ; \mathbb{Q})$ if an only if $\left[\tilde{c}_{i}(\widehat{X})\right] \in H^{2 i}(\widehat{X}, \widehat{M} ; \mathbb{Q})$.

As before, we suppress the change of $X$ to $\widehat{X}$, and simply assume that for any principal monodromy $\gamma_{i}$ around $E_{i}, \rho_{\mathbb{C}}\left(\gamma_{i}\right)$ is unipotent.

As a last reduction before the proof of the main theorem, let us consider a divisor $E_{i}$ whose principal monodromy is parabolic.

Proposition 5.2. For $\gamma_{i}$ parabolic, $X$ as above, then $\rho\left(\gamma_{i}\right) \in P S U(n, 1)$ is conjugate by a Cayley transform to an automorphism of $\mathcal{U}_{n}$ given by

$$
\mathcal{U}_{n} \ni(z, w) \rightarrow\left(z, w+t_{i}\right)
$$

for some $t_{i} \in \mathbb{R}$.

Proof. Referring back to equation (3.3), we see that $\rho\left(\gamma_{i}\right)$ is given, after a Cayley transform, by

$$
\mathcal{U}_{n} \ni(z, w) \rightarrow(z+a, w+t+2 \sqrt{-1}(z, a)+\sqrt{-1}(a, a))
$$

for some $a \in \mathbb{C}^{n-1}, t \in \mathbb{R}$, since in (3.3) we know $\lambda=1$, by proposition 3.1, and $A=I \in U(n-1)$, by the main lemma 4.1. It remains to show that $a=0 \in \mathbb{C}^{n-1}$. At a regular point of $E$ in $E_{i}$, let $\left(z_{1}, \ldots, z_{n-1}, \zeta\right)$ be local coordinates in which $E_{i}$ is given by $\zeta=0$. Writing the development map $D$ on the disk $z_{1}=\ldots=$ $z_{n-1}=0$ to $\mathcal{U}_{n}$, we now have that the pull-back $D^{*} H$, where $H$ is as before the height function on $\mathcal{U}_{n}$, is well-defined off $\zeta=0$. By the well-known fact that holomorphic sectional curvatures decrease on passage to complex submanifolds of Kähler manifolds, and the one-dimensional Schwarz(-Pick-Ahlfors) lemma, we get that

$$
\frac{\sqrt{-1}}{2} \frac{\partial D^{*} H \wedge \bar{\partial} D^{*} H}{D^{*} H^{2}} \leq \frac{\sqrt{-1}}{2} \partial \bar{\partial} \log \frac{1}{D^{*} H} \leq \frac{\sqrt{-1}}{2} \partial \bar{\partial} \log \log \left(\frac{1}{|\zeta|}\right)
$$

Integrating along any radial curve $\zeta=s e^{\sqrt{-1} \theta_{0}}$ from $s=r$ to $s=r_{o}>r$ to compute the arclength of this curve in the Poincaré metric as in the proof of 
proposition 3.1, we conclude

$$
\frac{1}{D^{*} H\left(r e^{\sqrt{-1} \theta_{0}}\right)} \geq \frac{\log \left(\frac{1}{r_{0}}\right)}{D^{*} H\left(r_{0} e^{\sqrt{-1} \theta_{0}}\right)} \cdot \frac{1}{\log \left(\frac{1}{r}\right)}
$$

and hence,

$$
\frac{1}{D^{*} H(\zeta)} \geq c \cdot \frac{1}{\log \left(\frac{1}{|\zeta|}\right)}
$$

for some positive constant $c$, provided $|\zeta|<r_{0}$.

Now let us fix $0<r<r_{0}$, and estimate the analytic continuation of $D$ along the circle $C_{r}$. Set $D\left(r e^{\sqrt{-1} \theta}\right)=\left(z_{1}(\theta), \ldots, z_{n-1}(\theta), w(\theta)\right) \in \mathcal{U}_{n}$. As observed earlier, $D\left(r e^{\sqrt{-1} \theta}\right)$ is a curve from $D(r)$ to $\rho\left(\gamma_{i}\right)(D(r))=(z(0)+a, w(r)+t+$ $2 \sqrt{-1}(z(r), a)+\sqrt{-1}(a, a))$. But we can estimate

$$
\int_{0}^{2 \pi}\left|\frac{d D}{d \theta}\left(r e^{\sqrt{-1} \theta}\right)\right|_{C H} d \theta \geq \int_{0}^{2 \pi} \frac{\left|\frac{d z}{d \theta}(\theta)\right|}{\sqrt{H\left(D\left(r e^{\sqrt{-1} \theta}\right)\right.}} d \theta
$$

where $\left|\frac{d z}{d \theta}(\theta)\right|=\sqrt{\sum_{i=1}^{n-1}\left|\frac{d z_{i}}{d \theta}(\theta)\right|^{2}}$. By (5.4), we get

$$
\int_{0}^{2 \pi} \frac{\left|\frac{d z}{d \theta}(\theta)\right|}{\sqrt{H\left(D\left(r e^{\sqrt{-1} \theta}\right)\right)}} d \theta \geq c^{\frac{1}{2}} \frac{1}{\log ^{\frac{1}{2}}\left(\frac{1}{r}\right)} \int_{0}^{2 \pi}\left|\frac{d z}{d \theta}(\theta)\right| d \theta .
$$

Finally, since $z(\theta)$ is a curve from $z(0)$ to $z(0)+a$, using Euclidean length in $\mathbb{C}^{n-1}$ gives

$$
\int_{0}^{2 \pi} \frac{\left|\frac{d z}{d \theta}(\theta)\right|}{\sqrt{H\left(D\left(r e^{\sqrt{-1} \theta}\right)\right)}} d \theta \geq c^{\frac{1}{2}} \frac{1}{\log ^{\frac{1}{2}}\left(\frac{1}{r}\right)}|a| .
$$

Substituting (3.2) into (5.7) and (5.6) gives

$$
\frac{C}{\log \frac{1}{2}\left(\frac{1}{r}\right)} \geq|a|,
$$

and letting $r \rightarrow 0$, we conclude $a=0$, proving proposition 5.2 .

\section{Completion of the Argument}

In this section we construct a holomorphic bundle $F$ over $X$ which is an extension of the pull-back $D^{*} T \mathbb{B}^{n}$ on $X \backslash E$ across $E \subset X$, and which admits an extension of the complex hyperbolic metric across all elliptic divisors $E_{j} \subset E$, and having only very mild singularities across the parabolic divisors $E_{i}$. This last 
part of the proof is a simple adaptation to the present situation of constructions of [11] and [23]. A set of comparisons as in the proof of proposition 5.1, and a check $\grave{a} l a[23]$ that the singular parts of the metric on $F$ do not contribute distributional terms to the Chern classes complete the proof of the main theorem.

Let 0 be a regular point on $E_{i}$, a parabolic component of $E$. Let $\left(z_{1}, \ldots, z_{n-1}, \zeta\right)$ be coordinates near 0 as in the proof of proposition 5.2, and let the development map (after Cayley transform) be $D: \Delta^{n-1} \times \Delta^{*} \rightarrow \mathcal{U}_{n}$, given by

$$
D(z, \zeta)=\left(f(z, \zeta), g(z, \zeta)+\frac{t_{i}}{2 \pi \sqrt{-1}} \log \zeta\right),
$$

where $t_{i}$ is as in proposition 5.2 , and $f(z, \zeta), g(z, \zeta)$ are single-valued on $\Delta^{n-1} \times$ $\Delta^{*}$.

Lemma 6.1. The functions $f(z, \zeta), g(z, \zeta)$ extend holomorphically across $\zeta=0$.

Proof. Consider the function $G(z, \zeta)=e^{\sqrt{-1} g(z, \zeta)}$ on $\Delta^{n-1} \times \Delta^{*}$. Since $\operatorname{Im}(g+$ $\left.\frac{t_{i}}{2 \pi \sqrt{-1}} \log \zeta\right)>0$, we have that

$$
|G(z, \zeta)| \leq \frac{1}{|\zeta|^{\frac{t_{i}}{2 \pi}}}
$$

Hence, $G(z, \zeta)$ is meromorphic across $\zeta=0$, and we may write

$$
G(z, \zeta)=\zeta^{\nu} \cdot G^{(\nu)}(z, \zeta)
$$

where $\nu \in \mathbb{Z}$, and $G^{(\nu)}$ is holomorphic across $\zeta=0$, and $G^{(\nu)} \neq 0$ in a neighborhood of some point $\left(z_{0}, 0\right) \in E_{i}$. But then, taking $\log$ of both sides of the equation

$$
e^{\sqrt{-1} g}=\zeta^{\nu} \cdot G^{(\nu)}
$$

shows that $\nu=0$, since otherwise $g$ would not be single-valued on $\Delta^{n-1} \times \Delta^{*}$. Then $G \neq 0$ near $\left(z_{0}, 0\right)$, which means that $g(z, \zeta)$ extends holomorphically across $E_{i}$ near $\left(z_{0}, 0\right)$, and hence everywhere. Since $D(z, \zeta) \in \mathcal{U}_{n}$, we have

$$
|f(z, \zeta)|^{2}<\operatorname{Im}(g(z, \zeta))+\frac{\left|t_{i}\right|}{2 \pi} \log \left(\frac{1}{|\zeta|}\right)
$$

or

$$
|f(z, \zeta)|^{2}<C\left(1+\log \left(\frac{1}{|\zeta|}\right)\right), C>>0 .
$$

This implies $f(z, \zeta)$ extends holomorphically across $E_{i}$, too.

Since we are assuming now that the principal monodromies are unipotent, if $E_{j}$ is an elliptic component of $E$, then the monodromy $\gamma_{j}$ verifies $\rho\left(\gamma_{j}\right)=I$. Then the development map $D$ itself extends across $E_{j}$ locally. Finally, at a general point $z_{\infty} \in E$, since $E$ is a divisor with normal crossings, we can find coordinates $\left(z_{1}, \ldots, z_{k}, w_{1}, \ldots, w_{\ell}, \zeta_{1}, \ldots, \zeta_{d}\right)$, where $w_{j}=0, j=1, \ldots, \ell$, are the 
elliptic divisor components passing through $z_{\infty}$, and $\zeta_{i}=0, i=1, \ldots, d$, are the parabolic divisor components through $z_{\infty}$. Fixing a basepoint $z_{0} \in \Delta^{n-d} \times\left(\Delta^{*}\right)^{d}$ we see that all $\rho\left(\gamma_{i}\right), i=1, \ldots, d$, must commute with each, and hence fix the same point on the boundary of $\mathbb{B}^{n}$. Passing to the Cayley transform $\mathcal{U}_{n}$, the same method as in the proof of lemma 6.1 shows:

Corollary 6.1. Near $z_{\infty}$, we may write

$$
D(z, w, \zeta)=\left(f(z, w, \zeta), g(z, w, \zeta)+\sum_{i=1}^{d} \frac{t_{i}}{2 \pi \sqrt{-1}} \log \zeta_{i}\right),
$$

where $f, g$ are holomorphic on all of $\Delta^{n}$.

Fixing $z_{0}, w_{0}$, and $\left(\zeta_{0}\right)_{j}, j \neq i$, and letting $\zeta_{i}=r_{i} \in(0,1) \rightarrow 0$, that

$$
D\left(z_{0}, w_{0},\left(\zeta_{0}\right)_{1}, \ldots, r_{i}, \ldots,\left(\zeta_{0}\right)_{d}\right) \in \mathcal{U}_{n},
$$

for all $r_{i} \rightarrow 0$, we get the following corollary.

Corollary 6.2. In corollary 6.1, the coefficients $t_{i}$ are all positive.

The development map $D: X \backslash E \rightarrow \mathbb{B}^{n}$ allows us to pull back the holomorphic tangent bundle $T \mathbb{B}^{n}$, together with the differential $d D_{*}:\left.T X\right|_{X \backslash E} \rightarrow D^{*} T \mathbb{B}^{n}$. This is obviously true on the universal covering $\widetilde{X \backslash E}$ of $X \backslash E$, but then the bundle and differential $d D_{*}$ are both equivariant with respect to the actions of $\pi_{1}\left(X \backslash E, \tilde{z}_{0}\right)$ on $\widehat{X \backslash E}$ and $\rho\left(\pi_{1}\left(X \backslash E, \widetilde{z}_{0}\right)\right)$ on $\mathbb{B}^{n}$, allowing the construction to be pushed down to $X \backslash E$. $D^{*} T \mathbb{B}^{n}$ obviously extends across all points of $E$ contained only in elliptic components of $E$.

We next would like to extend both $\left.T X\right|_{X \backslash E}$ and $D^{*} T \mathbb{B}^{n}$ across the parabolic components. First, define $T_{\text {par }} X$ to be the bundle of vector fields tangent to $E_{\text {par }}$ along $E_{\text {par }}:=\cup_{E_{i} \text { parabolic }} E_{i}$, explicitly spanned by the $\frac{\partial}{\partial z_{s}}, \frac{\partial}{\partial w_{j}}, \zeta_{i} \frac{\partial}{\partial \zeta_{i}}$ in the local coordinates given above. For $D^{*} T \mathbb{B}^{n}$, following Mumford, we define a holomorphic bundle $F$ extending it to $X$ by the condition that its holomorphic sections $\Gamma\left(\Delta^{n}, \mathcal{O}(F)\right)$ are those holomorphic sections of $D^{*} T \mathbb{B}^{n}$ over $\Delta^{n-d} \times\left(\Delta^{*}\right)^{d}$ which are bounded in the pull back of the complex hyperbolic metric on $D^{*} T \mathbb{B}^{n}$.

Lemma 6.2. $F$ is a holomorphic vector bundle. The differential of $D$ extends to give a holomorphic bundle homomorphism $d D_{*}: T_{\text {par }} X \rightarrow F$, which is an isomorphism on $X \backslash E$.

Proof. We check that $F$ is a vector bundle near $z_{\infty} \in E_{\text {par }}$. We write $D$ : $\Delta^{n-d} \times\left(\Delta^{*}\right)^{d} \rightarrow \mathcal{U}_{n}$ as above. We claim that a basis for $F$ over $\Delta^{n}$ is given by $Z_{1}, \ldots, Z_{n-1}, W$, where $Z_{j}(z, \zeta)=\left.\frac{\partial}{\partial z_{j}}\right|_{(z, w)=D(z, \zeta)}, W(z, \zeta)=\left.\frac{\partial}{\partial w}\right|_{(z, w)=D(z, \zeta)}$. Note that since $D$ is not single-valued, $Z_{j}, W$ denote equivalence classes of vectors, where the equivalence class, e.g., $\left[\left.\frac{\partial}{\partial z_{j}}\right|_{(z, w)}\right]=\left\{\left.\frac{\partial}{\partial z_{j}}\right|_{\left(z, w+\sum_{i=1}^{d} m_{i} t_{i}\right)} \mid m_{i} \in \mathbb{Z}\right\}$. The 
complex hyperbolic length of $Z_{j}$ is independent of the representative. Direct calculation shows

$$
\left|Z_{j}\right|_{C H}^{2}=\frac{\left|f_{j}\right|^{2}}{D^{*} H^{2}}+\frac{1}{D^{*} H} \leq C,
$$

and

$$
|W|_{C H}^{2}=\frac{1}{D^{*} H^{2}} \leq C,
$$

verifying that these are sections of $F$. To prove that they are a basis of $F$, we consider a section $Z=\sum a_{j}(z, \zeta) Z_{j}+b(z, \zeta) W$, where $a_{j}, b$ are holomorphic on $\Delta^{n-d} \times\left(\Delta^{*}\right)^{d}$, with $|Z|_{C H}^{2} \leq C$, we deduce

$$
\frac{|a|^{2}}{D^{*} H} \leq|Z|_{C H}^{2} \leq C,
$$

so that the coefficients $a_{j}$ extend holomorphically to $\Delta^{n}$. Similarly, we now know

$$
|b W|_{C H}^{2}=\frac{|b|^{2}}{D^{*} H^{2}} \leq C,
$$

showing that $b$ extends holomorphically to $\Delta^{n}$ as well, proving the $Z_{j}, W$ are a local basis for $F$.

To show that the differential $d D_{*}$ extends holomorphically across $E_{\text {par }}$, using the same local coordinates as above, we compute

$$
\begin{aligned}
d D_{*}\left(\frac{\partial}{\partial z_{j}}\right) & =\sum \frac{\partial f_{s}}{\partial z_{j}} Z_{s}+\frac{\partial g}{\partial z_{j}} W, \\
d D_{*}\left(\frac{\partial}{\partial w_{j}}\right) & =\sum \frac{\partial f_{s}}{\partial w_{j}} Z_{s}+\frac{\partial g}{\partial w_{j}} W, \\
d D_{*}\left(\zeta_{i} \frac{\partial}{\partial \zeta_{i}}\right) & =\sum \zeta_{i} \frac{\partial f_{s}}{\partial \zeta_{i}} Z_{s}+\left(\zeta_{i} \frac{\partial g}{\partial \zeta_{i}}+\frac{t_{i}}{2 \pi \sqrt{-1}}\right) W,
\end{aligned}
$$

Since all the coefficients of $Z_{j}, W$ on the right hand side of (6.5) are holomorphic on $\Delta^{n}, d D_{*}: T_{p a r} X \rightarrow F$ as claimed.

In what follows, we will no longer have to distinguish between the coordinates $z_{j}$ and $w_{k}$ in $\Delta^{n-d} \times\left(\Delta^{*}\right)^{d}$, so we will drop mention of the $w_{k}$ 's.

With these bundles in hand, we can make a series of comparisons. Note that there is an inclusion bundle map $\iota: T_{p a r} X \rightarrow T X$. All the bundles will be assumed to have the same metric induced from the complex hyperbolic metric on $\mathbb{B}^{n}$ off of $E$. We extend this to a hermitian metric on each of these bundles 
differing from the complex hyperbolic metric only within any small open neighborhood of $E$. Then $\left[\tilde{c}_{i}\left(T_{p a r} X\right)-\tilde{c}_{i}(T X)\right]$, and $\left[\tilde{c}_{i}\left(T_{p a r} X\right)-\tilde{c}_{i}(F)\right]$ are both in $H^{2 i}(X, M ; \mathbb{Q})$. Thus, the main theorem reduces finally to the following lemma:

Lemma 6.3. $\left[\tilde{c}_{i}(F)\right] \in H^{2 i}(X, M ; \mathbb{R})$ vanishes.

Proof. We consider two metrics on $F$ : the first is a metric $h_{X}$ which will be smooth on $X$, and agreeing with the pull-back $D^{*} h_{C H}$ of the complex hyperbolic metric off a small neighborhood of $E_{p a r}$, and the second is $h=D^{*} h_{C H}$ itself. This latter, is, of course, singular along $E_{p a r}$. Recall the definitions from [23]:

Definition 6.4. A smooth $p$-form $\eta$ on $X \backslash E$ has Poincaré growth on $X \backslash E$ if in any polydisk neighborhood of $z_{\infty}$ as above, and any tangent vectors $v_{1}, \ldots, v_{p}$ on $\Delta^{n}$, we have an estimate $\left|\eta\left(v_{1}, \ldots, v_{p}\right)\right|^{2} \leq C \prod\left|v_{j}\right|_{P}^{2}$, where $d s_{P}^{2}$ is any local Poincaré metric comparable to

$$
d s_{P}^{2}=\sum_{j}\left|d z_{j}\right|^{2}+\sum_{i} \frac{\left|d \zeta_{i}\right|^{2}}{\left|\zeta_{i}\right|^{2} \log ^{2} \frac{1}{\left|\zeta_{i}\right|^{2}}}
$$

on $\Delta^{n-d} \times\left(\Delta^{*}\right)^{d}$. The $p$-form $\eta$ is good if $\eta$ and $d \eta$ have Poincaré growth.

Definition 6.5. A hermitian metric $h$ on $F$ over $X \backslash E$ is $\operatorname{good}$ on $X$ if in any polydisk neighborhood of $z_{\infty}$ as above, and if $h_{i, \bar{j}}=h\left(e_{i}, e_{j}\right)$ in a local basis for $F$ on $\Delta^{n}$, then
i) $\left|h_{i, \bar{j}}\right|,\left(\operatorname{det} h_{i, \bar{j}}\right)^{-1} \leq C\left(\sum_{i=1}^{d} \log \left|\zeta_{i}\right|\right)^{2 k}$, for some $C>0, k \geq 0$,
ii) the 1-forms $\left(\partial h \cdot h^{-1}\right)_{i, \bar{j}}$ are good in the sense of definition 6.4.

We verify that $D * h_{C H}$ is good on $F$ by calculating in $\mathcal{U}_{n}$ and pulling back to $\Delta^{n-d} \times\left(\Delta^{*}\right)^{d}$. We calculate in the basis $Z_{j}, W$ of $F$, which is equivalent to calculating in the basis $\frac{\partial}{\partial z_{j}}, \frac{\partial}{\partial w}$ of $T \mathcal{U}_{n}$. On $\mathcal{U}_{n}$, the components of $h_{C H}$ are given explicitly as

$$
\begin{aligned}
& h_{C H}\left(\frac{\partial}{\partial z_{j}}, \frac{\partial}{\partial z_{l}}\right)=\frac{\delta_{j, \bar{\ell}}}{H}+\frac{\bar{z}_{j} z_{l}}{H^{2}}, j, l=1, \ldots, n-1, \\
& h_{C H}\left(\frac{\partial}{\partial z_{j}}, \frac{\partial}{\partial w}\right)=\frac{\bar{z}_{j}}{2 \sqrt{-1} H^{2}}, j=1, \ldots, n-1, \\
& h_{C H}\left(\frac{\partial}{\partial w}, \frac{\partial}{\partial w}\right)=\frac{1}{4 H^{2}},
\end{aligned}
$$


which implies that on $\Delta^{n-d} \times\left(\Delta^{*}\right)^{d}$ we have

$$
\begin{aligned}
& h\left(Z_{j}, Z_{l}\right)=\frac{\delta_{j, \bar{\ell}}}{D^{*} H}+\frac{\bar{f}_{j} f_{l}}{H^{2}}, j, l=1, \ldots, n-1, \\
& h\left(Z_{j}, W\right)=\frac{\bar{f}_{j}}{2 \sqrt{-1} D^{*} H^{2}}, j=1, \ldots, n-1, \\
& h(W, W)=\frac{1}{4 D^{*} H^{2}},
\end{aligned}
$$

where $f_{j}$ is as in corollary 6.1 . Together with $(\operatorname{det} h)^{-1}=C \cdot H^{n+1}$ this verifies part $i$ ) of definiton 6.5. For part ii), we first observe that any entry of $h^{-1}$ is bounded by $C D^{*} H$. From (6.7), we see that $\left|\frac{\partial h_{s, \bar{t}}}{\partial z_{j}}\right| \leq \frac{C}{D^{*} H^{2}}$ and thus

$$
\left|<\partial h \cdot h^{-1}, \frac{\partial}{\partial z_{j}}>\right| \leq \frac{C}{D^{*} H} \leq C^{\prime} .
$$

Similarly, $\left|<\partial h, \frac{\partial}{\partial \zeta_{i}}>\right| \leq \frac{C}{D^{*} H^{2}\left|\zeta_{i}\right|}$, and $\left|\frac{\partial h_{s, \bar{t}}}{\partial \zeta_{i}}\right| \leq \frac{C}{D^{*} H^{2}\left|\zeta_{i}\right|}$ and

$$
\left|<\partial h \cdot h^{-1}, \frac{\partial}{\partial \zeta_{i}}>\right| \leq \frac{C}{D^{*} H\left|\zeta_{i}\right|},
$$

showing that $\partial h \cdot h^{-1}$ has Poincaré growth. The same applies to $\bar{\partial} h \cdot h^{-1}$. As to $d\left(\partial h \cdot h^{-1}\right)$, we refer to $(6.7)$ to see that

$$
\begin{aligned}
& \left|\frac{\partial^{2} h_{s, \bar{t}}}{\partial z_{j} \partial \bar{z}_{l}}\right| \leq \frac{C}{D^{*} H^{2}} \\
& \left|\frac{\partial^{2} h_{s, \bar{t}}}{\partial z_{j} \partial \bar{\zeta}_{i}}\right| \leq \frac{C}{D^{*} H^{3}\left|\zeta_{i}\right|} \\
& \left|\frac{\partial^{2} h_{s, \bar{t}}}{\partial \zeta_{i} \partial \bar{\zeta}_{k}}\right| \leq \frac{C}{D^{*} H^{3}\left|\zeta_{i}\right|\left|\zeta_{k}\right|}
\end{aligned}
$$

Hence, we get

$$
\begin{aligned}
& \left|\partial \bar{\partial} h \cdot h^{-1}\left(\frac{\partial}{\partial z_{j}}, \frac{\partial}{\partial \bar{z}_{k}}\right)\right|^{2} \leq \frac{C}{D^{*} H^{2}}, \\
& \left|\partial \bar{\partial} h \cdot h^{-1}\left(\frac{\partial}{\partial z_{j}}, \frac{\partial}{\partial \bar{\zeta}_{i}}\right)\right|^{2} \leq \frac{C}{D^{*} H^{4}\left|\zeta_{i}\right|^{2}} \leq \frac{C}{D^{*} H^{2}\left|\zeta_{i}\right|^{2} \log \left|\zeta_{i}\right|^{2}}, \\
& \left|\partial \bar{\partial} h \cdot h^{-1}\left(\frac{\partial}{\partial \zeta_{i}}, \frac{\partial}{\partial \bar{\zeta}_{k}}\right)\right|^{2} \leq \frac{C}{\left|\zeta_{i}\right|^{2}\left|\zeta_{k}\right|^{2} D^{*} H^{4}} \leq \frac{C}{\left|\zeta_{i}\right|^{2} \log ^{2}\left|\zeta_{i}\right|^{2} \cdot\left|\zeta_{k}\right|^{2} \log ^{2}\left|\zeta_{k}\right|^{2}}
\end{aligned}
$$


and hence $\partial \bar{\partial} h \cdot h^{-1}$ is of Poincaré growth. Since the product of forms with Poincaré growth is again of Poincaré growth, this shows that $d\left(\partial h \cdot h^{-1}\right)$ is of Poincaré growth, and so $\partial h \cdot h^{-1}$ is good on $\Delta^{n-d} \times\left(\Delta^{*}\right)^{d}$, and therefore $h$ is good on $F$ over $\Delta^{n-d} \times\left(\Delta^{*}\right)^{d}$.

Let now $A, A^{o}$ denote the connection forms for the canonical hermitian connections on $F$ with respect to $h_{X}, h$, respectively, over $\Delta^{n-d} \times\left(\Delta^{*}\right)^{d}$, and similarly, let $\Omega, \Omega^{o}$ be the corresponding curvature forms. For any symmetric, $A d-$ invariant polynomial $P(\Omega, \ldots, \Omega)$ homogeneous of degree $\nu$, we have a transgression $(2 \nu-1)$-form $T P\left(A-A^{o}, \Omega, \Omega^{o}\right)$ polynomial in its arguments, so that on $\Delta^{n-d} \times\left(\Delta^{*}\right)^{d}$ we have

$$
P(\Omega, \ldots, \Omega)-P\left(\Omega^{o}, \ldots, \Omega^{o}\right)=d T P\left(A-A^{o}, \Omega, \Omega^{o}\right) .
$$

Since the component forms of $\Omega^{o}, A^{o}$ are good on $\Delta^{n-d} \times\left(\Delta^{*}\right)^{d}$, the forms $P\left(\Omega^{o}, \ldots, \Omega^{o}\right), T P\left(A-A^{o}, \Omega, \Omega^{o}\right)$ and $d T P\left(A-A^{o}, \Omega, \Omega^{o}\right)$ are all locally integrable across $\prod \zeta_{i}=0$, and so define currents $\left[P\left(\Omega^{o}, \ldots, \Omega^{o}\right)\right]$, $\left[T P\left(A-A^{o}, \Omega, \Omega^{o}\right)\right]$ and $\left[d T P\left(A-A^{o}, \Omega, \Omega^{o}\right)\right]$ on $X$. The point of good forms is proposition 1.2 of [23]: for a good form $\eta,[d \eta]=d[\eta]$. Thus, the characteristic forms satisfy the equation of currents

$$
P(\Omega, \ldots, \Omega)-\left[P\left(\Omega^{o}, \ldots, \Omega^{o}\right)\right]=d\left[T P\left(A-A^{o}, \Omega, \Omega^{o}\right)\right]
$$

on $X$. In our case, the currents $P(\Omega, \ldots, \Omega)-\left[P\left(\Omega^{o}, \ldots, \Omega^{o}\right)\right]$ and $T P(A-$ $\left.A^{o}, \Omega, \Omega^{\circ}\right)$ are compactly supported in any small open neighborhood of $E$. (Notice that $A-A^{o}$ is globally defined, and supported near $E$.) More specifically, when $P(\Omega, \ldots, \Omega)$ gives a renormalized Chern form $\tilde{c}_{i}(\Omega)$, or a polynomial of such, we obtain $P\left(\Omega^{o}, \ldots, \Omega^{o}\right) \equiv 0$ on $\Delta^{n-d} \times\left(\Delta^{*}\right)^{d}$, and so $\left[P\left(\Omega^{o}, \ldots, \Omega^{o}\right)\right] \equiv 0$ on $X$. Thus we are left in such a case with the equation of currents $P(\Omega, \ldots, \Omega)=$ $d\left[T P\left(A-A^{o}, \Omega, \Omega^{o}\right)\right]$ on $X$, which implies the class of $P(\Omega, \ldots, \Omega) \in H^{2 \nu}(X, M ; \mathbb{R})$ is 0 , proving lemma 6.3 and hence Theorem 1.2 and Theorem 1.1.

\section{Open Questions; the Real Analogue}

There are many questions left open by the present work. In general, it is still unknown what the relationship is between the renormalized Chern classes of [7] and, for example, index problems on pseudonconvex manifolds. One indication of such a relationship is given by [13], and unpublished calculations of C. Epstein show that renormalizability of combinations of Chern classes occurring in Riemann-Roch theorems is correlated with spectral behavior of the kinds of operators studied in [13]. Encouraging results in this direction have appeared very recently in the Stanford thesis of P. Albin [1]. 
Similar problems arise for conformally compact manifolds $X$ whose boundaries $M=\partial X$ are conformally flat and the Pontrjagin classes of $X$. In this case one cannot expect the integrals of such forms over $X$ to be rational, in general, because of the relationship to the volumes of hyperbolic three manifolds [12], [21] and because there is no canonical way to fill in the boundary manifold $M$, as there is in the complex case (up to blowing up). A natural class of $X$ to consider would be $X$ admitting a conformally compact Einstein metric. This would be interesting in that one does not know when such a conformally flat $M$ should admit a fill-in $X$ with such a metric.

The method of renormalization of [7] is essentially algebraic, and renormalizes the characteristic class $c_{1}(X)^{n}$ to 0 . In the case of Kähler-Einstein manifolds, this class is proportional to the volume form. Recent geometric cut-off renormalizations of volume of conformally compact [16] and Kähler-Einstein manifolds [27] are directly in the spirit of physical applications. These suggest that all Chern classes may be renormalized in a similar fashion, and with non-trivial contribution for the $c_{1}^{n}$.

Finally, the present work avoids explicit evaluation of the renormalized characteristic numbers, in particular, their evaluation by residue formulas. Examples in [24] confirm that, although the evaluation can be made locally along $E$, what has to be evaluated is global (coming from $M$ by analytic continuation, and not just local geometry of $E$ ). In the present context this appears in the bundle $F$ of section 6 . The only residue computed here is that in section 6 . coming from [23]. The intermediate reductions use weak forms of the Thom-Porteous relations. Residue formulas can be expected to work in those cases where the degeneracy loci of [15], e.g., associated to bundle maps such as $d D_{*}: T_{p a r} X \rightarrow F$ of section 6. are of the correct dimension [18].

\section{REFERENCES}

[1] P. Albin, Thesis, Stanford University, 2005.

[2] M. Artin, Algebraic approximation of structures over complete local rings, Publ. Math. IHES 36 (1969), 23-58.

[3] A. Borel, Compact Clifford-Klein forms of symmetric spaces, Topology 2 (1963), 111122.

[4] R. Bott and S.S. Chern, Holomorphic vector bundles and the equidistribution of the zeroes of their holomorphic sections, Acta Math. 114 (1965), 71-112.

[5] D. Burns, Analytic continuation and development maps in CR and complex-hyperbolic geometry, to appear.

[6] D. Burns and C. Epstein, A global invariant of 3-dimensional CR-manifolds, Invent. Math. 92 (1988), 333-348.

[7] -----, Characteristic numbers of bounded domains, Acta Math. 164 (1990), 29-71.

[8] D. Burns and R. Mazzeo, On the geometry of cusps for $S U(n, 1)$, in Manifolds and Geometry, P. de Barolomeis, F. Tricerre and E. Vesentini, eds., Symposia Matematica XXXVI, Cambridge, Cambridge University Press (1996). 
[9] S.-Y. Cheng and S.-T. Yau, On the existence of a complete Kähler metric on noncompact complex manifolds and the regularity of Fefferman's equation, Comm. Pure Appl. Math. 33 (1980), 507-544.

[10] S.S. Chern and J. Moser, Real hypersurfaces in complex manifolds, Acta Math., 133 (1974), 219-271.

[11] M. Cornalba and P.A. Griffiths, Analytic cycles and vector bundles on non-compact algebraic varieties, Invent. Math. 28 (1975), 1-105.

[12] X. Dai, Eta invariant, conformally flat structures and hyperbolic structures, arXiv: math.DG/0106172.

[13] H. Donnelly and C. Fefferman, $L^{2}$-cohomology and index formula for the Bergman metric, Ann. Math. (2) 118 (1983), 593-618.

[14] C. Fefferman, Monge-Ampère equations, the Bergman kernel and the geometry of pseudoconvex domains, Ann. Math. (2) 103 (1976), 395-416.

[15] W. Fulton, Intersection Theory, Ergeb. Math. Grenzgeb., 3. Fol., Bd. 2, Berlin, SpringerVerlag (1984).

[16] C.R. Graham, Volume and area renormalizations for conformally compact Einstein metrics, Rend. Circ. Mat. Palermo (2) Suppl. 63 (2000), 31-42.

[17] P.A. Griffiths, Entire holomorphic mappings of one and several complex variables, Ann. Math. Studies 99, Princeton, Princeton University Press (1976).

[18] F.R. Harvey and H.B. Lawson, Jr., Geometric residue theorems, Amer. J. Math. 117 (1995), 829-873.

[19] H. Hironaka, Resolution of singularities of an algebraic variety over a field of characteristic zero, I, II, Ann. Math. (2) 79 (1964), 109-203 and 205-326.

[20] J. Lee and R. Melrose, Boundary behavior of the complex Monge-Ampère equation, Acta Math. 148 (1982), 159-192.

[21] D.D. Long and A.W. Reid, On the geometric boundaries of hyperbolic four manifolds, Geom. \& Top. 4 (2000), 171-178.

[22] D. Mumford, The topology of normal singularities of an algebraic surface and a criterion for simplicity, Publ. Math. IHES 9 (1961), 5-22.

[23] -----, Hirzebruch's proportionality principle in the non-compact case, Invent. Math. 42 (1977), 239-272.

[24] J.-S. Ryu, Development Mapping on CR-manifolds, Thesis, University of Michigan, (1991).

[25] R. Schoen and S.-T. Yau, Conformally flat manifolds, Kleinian groups and scalar curvature, Invent. Math. 92 (1988), 47-71.

[26] A. Selberg, On discontinuous groups in higher dimensional symmetric spaces, in Contributions to Function Theory, Tata Institute of Fundamental Research, Bombay, 1960.

[27] N. Seshadri, Volume renormalization for complete Einstein-Kähler metrics, arXiv: math.DG/0404455.

[28] S.-T. Yau, A general Schwarz lemma for Kähler manifolds, Am. J. Math. 100 (1978), 197-203.

D. Burns

University of Michigan, Ann Arbor

MI 48109-1109 USA

E-mail:dburns@umich.edu

J.-S. Ryu

Hongik University, Seoul 121-791 Korea

E-mail:jsryu@math.hongik.ac.kr 